\title{
New Type of Quantum Criticality in the Pyrochlore Iridates
}

\author{
Lucile Savary, ${ }^{1, *}$ Eun-Gook Moon, ${ }^{1}$ and Leon Balents ${ }^{2}$ \\ ${ }^{1}$ Department of Physics, University of California, Santa Barbara, California 93106-9530, USA \\ ${ }^{2}$ Kavli Institute for Theoretical Physics, University of California, \\ Santa Barbara, California 93106-4030, USA
}

(Received 6 May 2014; revised manuscript received 1 August 2014; published 13 November 2014)

\begin{abstract}
Magnetic fluctuations and electrons couple in intriguing ways in the vicinity of zero-temperature phase transitions-quantum critical points-in conducting materials. Quantum criticality is implicated in nonFermi liquid behavior of diverse materials and in the formation of unconventional superconductors. Here, we uncover an entirely new type of quantum critical point describing the onset of antiferromagnetism in a nodal semimetal engendered by the combination of strong spin-orbit coupling and electron correlations, and which is predicted to occur in the iridium oxide pyrochlores. We formulate and solve a field theory for this quantum critical point by renormalization group techniques and show that electrons and antiferromagnetic fluctuations are strongly coupled and that both these excitations are modified in an essential way. This quantum critical point has many novel features, including strong emergent spatial anisotropy, a vital role for Coulomb interactions, and highly unconventional critical exponents. Our theory motivates and informs experiments on pyrochlore iridates and constitutes a singular realistic example of a nontrivial quantum critical point with gapless fermions in three dimensions.
\end{abstract}

DOI: 10.1103/PhysRevX.4.041027

\section{INTRODUCTION}

Antiferromagnetic quantum critical points (QCPs) are controlled by the interactions between electrons and magnetic fluctuations $[1,2]$. In three-dimensional metals with a Fermi surface, it is believed, in most cases, to be sufficient to consider a theory containing the magnetic-order parameter only, with the Fermi surface included solely by its induced Landau damping. This leads, following Hertz $[3,4]$, to mean-field behavior. In two dimensions, the electronic Fermi surface and order parameter are strongly coupled, a fact which may be related to high-temperature superconductivity and associated phenomena. This problem is highly nontrivial and still an active research topic [5-8].

In this paper, we uncover a new antiferromagnetic QCP that is strongly coupled in three dimensions, engendered by spin-orbit coupled electronic structure. We consider a quadratic band touching at the Fermi energy, as in the inverted band-gap material $\mathrm{HgTe}$, but having in mind the strongly correlated family of iridium oxide pyrochlores [9-12]. The latter have chemical formula $\mathrm{A}_{2} \mathrm{Ir}_{2} \mathrm{O}_{7}$, and an antiferromagnetic phase transition indeed occurs both as a function of temperature and at zero temperature with varying chemical pressure (ionic radius of A) [13,14].

\footnotetext{
*To whom all correspondence should be addressed. lsavary@physics.ucsb.edu

Published by the American Physical Society under the terms of the Creative Commons Attribution 3.0 License. Further distribution of this work must maintain attribution to the author(s) and the published article's title, journal citation, and DOI.
}

Subject Areas: Strongly Correlated Materials

We show that the replacement of the Fermi surface by a point Fermi node alters the physics in an essential way, suppressing screening of the Coulomb interaction and allowing the order-parameter fluctuations to affect all the low-energy electrons. These two facts lead to a strongly-coupled quantum critical point.

The nodal nature of the Fermi point, happily, also enables a rather complete analysis of the problem, which we present here, using the powerful renormalization group (RG) technique. This is because both the collective orderparameter fluctuations and the low-energy quasiparticles have their excitation minima at a single point in momentum space (here at $\mathbf{k}=\mathbf{0}$ ), unlike the case of criticality in a metal, where the electronic quasiparticles have their minima at an extended Fermi surface. The complete theory we present is in sharp contrast to the strongly coupled Fermi surface problem in two dimensions, which remains only partially understood and controversial. We fully and explicitly describe a stable nontrivial quantum critical RG fixed point, including logarithmic corrections, which are also calculated systematically. The ability to systematically calculate logarithmic corrections may be important for future comparisons to experiment.

The results are summarized by the phase diagram, Fig. 1. The QCP we describe separates two phases. On the nonmagnetic side of the transition, the quadratically dispersing electrons near the Fermi node remain but are strongly renormalized by the long-range Coulomb interactions. This non-Fermi liquid semimetal is the LuttingerAbrikosov-Beneslavskii (LAB) phase of Ref. [10]. On the magnetic side of the transition, the quadratic band touching 


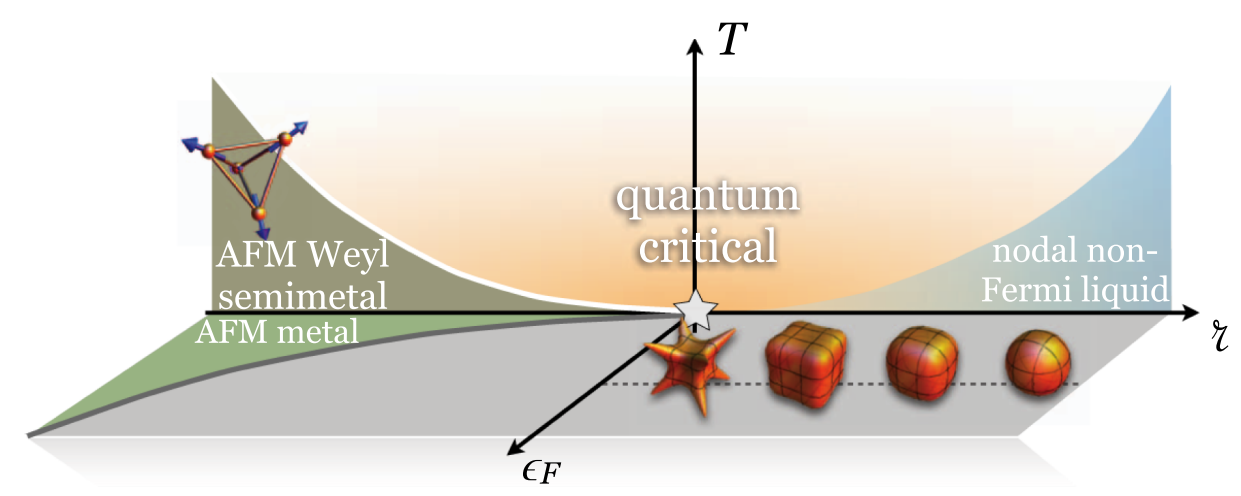

FIG. 1. Quantum critical point (QCP) and quantum criticality driven by the onset of "all-in-all-out" (AIAO) magnetism. For $r \geq r_{c}$ (in this figure the star indicates $r_{c}$ ), the nodal non-Fermi liquid (or "Luttinger-Abrikosov-Beneslavskii" [10]) phase occurs at $T=0$, with a quadratic Fermi node, while antiferromagnetic (AFM) all-in-all-out ordering occurs for $r<r_{c}$, with the quadratic node replaced by linear Weyl points - a Weyl semimetal. The quantum critical regime occurs at $T>0$ around $r=r_{c}$. Note that the quantum critical-AFM boundary (thick white line) is a true (continuous Ising) phase transition. The $\epsilon_{F}$ axis represents the Fermi energy and parametrizes electron or hole doping. The three-dimensional (orange) surfaces represent the shapes of the corresponding Fermi surfaces at small doping - the increased anisotropy is apparent as one moves towards the QCP. The phase transition denoted by the thick gray line is expected to exhibit critical properties appropriate to a $\mathbf{q}=\mathbf{0}$ order parameter coupled to a Fermi surface, as in the Hertz formulation [3], though subject to the usual uncertainties regarding the theory of that problem [5-7].

is split into a set of eight linearly dispersing "Weyl" points by the magnetic-order parameter, forming a Weyl semimetal state. The electronic dispersions in both phases are shown in Figs. 2(c) and 2(a). The results of this paper apply under the condition of charge neutrality (i.e., for undoped materials). Doping introduces a second tuning parameter, and the expected behavior in this enlarged phase space, based on physical and scaling arguments, is shown in the horizontal plane of Fig. 1.

As usual, scaling laws apply in the critical fan of the QCP (experimental implications are discussed further in Sec. V C). Furthermore, the pyrochlore quantum critical point has a remarkable symmetry structure. We find that, unlike at most classical and quantum phase transitions, rotational invariance is strongly broken in the critical theory: The fixed point "remembers" the cubic anisotropy of space (and indeed takes it to an extreme limit, as explained further below). Compensating for the absence of spatial rotational invariance is, however, an emergent $S O(3)$ invariance of the critical field theory, which is a purely internal symmetry and unrelated to spatial rotations. The anisotropy in real space can, for example, be studied by using light carrier doping as a probe. Then, we predict the formation of "spiky" Fermi surfaces, if the system is close to the undoped QCP and the doping is sufficiently small, as seen in Fig. 1.

\section{MODEL AND PHASES}

\section{A. All-in-all-out order}

To proceed with the analysis, we couple the electrons to an Ising magnetic order parameter $\phi$. This corresponds, for the pyrochlore iridates, to the translationally invariant all-in-all-out antiferromagnetic state (see inset in Fig. 1), for which there is considerable evidence [15-17]. Some understanding of this order follows from a local view of the magnetism. In the pyrochlore iridates, a single hole resides on each $\mathrm{Ir}^{4+}$ ion. In the localized (large Hubbard $U$ limit), this forms a Kramers doublet, whose orbital degeneracy is generically split by some combination of spin-orbit coupling and noncubic crystal fields. In a simple view based on spin-orbit coupling alone, this doublet is the famous $J_{\text {eff }}=$ $1 / 2$ state, but for our considerations, the precise composition of the doublet does not matter. The form of allowed nearest-neighbor interactions between dipolar Kramers doublets on the pyrochlore lattice follows from symmetry alone and has been discussed in many references (see, for example, Ref. [18]). The simplest amongst four allowed couplings, dominant in many materials, is the Ising term

$$
H_{\text {Ising }}=J_{z z} \sum_{\langle i j\rangle} \mathrm{S}_{i}^{z} \mathrm{~S}_{j}^{z}
$$

where $\mathrm{S}_{i}^{z}$ is the component of a spin-1/2 operator on site $i$ along its local $\langle 111\rangle$ Ising axis, which orients from the center of a I ("up") sublattice tetrahedron to the neighboring II ("down") sublattice tetrahedron, along a line containing the site $i$. This Hamiltonian, for $J_{z z}<0$, describes nearestneighbor interactions between rare earth spins in the socalled spin-ice materials, $\mathrm{Ho}_{2} \mathrm{Ti}_{2} \mathrm{O}_{7}$ and $\mathrm{Dy}_{2} \mathrm{Ti}_{2} \mathrm{O}_{7}$. It also describes the dominant interaction in quantum spinice compounds, such as $\mathrm{Yb}_{2} \mathrm{Ti}_{2} \mathrm{O}_{7}$ [18]. In the iridates, "antiferromagnetic" coupling is expected from the usual $d$-electron superexchange, which corresponds, because of the choice of local quantization axis, to $J_{z z}<0$ above. With this sign, in the local basis, the ground states are obviously simple Ising ferromagnets, with $\mathrm{S}_{i}^{z}=+1 / 2$ or $\mathrm{S}_{i}^{z}=-1 / 2$, independently of $i$. The actual magnetic moments in the two cases orient all-in or all-out of each 
tetrahedra, with zero total moment (see Fig. 1). We refer to this as AIAO antiferromagnetic order.

The above picture is obviously deficient insofar as the pyrochlore iridates are conducting, so the Ir electrons are not localized. The fact that a simple localized picture nevertheless describes the observed order is an indication that these materials are in an intermediate correlation regime. A local moment description of magnetic order and excitations is not uncommon in this situation and has been successful, for example, in many iron pnictides [19] and in other iridates $[20,21]$. We emphasize, however, that it is not important for our treatment, which relies only on symmetries.

\section{B. Electronic structure and phases}

We must couple the AIAO magnetic order and its fluctuations to the extended electronic states. Because of the time-reversal and inversion symmetries of the paramagnetic state, electron bands are twofold degenerate, so that band touching necessitates a minimal four-band model. Therefore, the Hamiltonian is expressed in terms of fourcomponent fermion operators $\psi, \psi^{\dagger}$, in addition to the order parameter $\phi$ and the electrostatic field $\varphi$, which mediates the Coulomb interactions. The action is

$$
\begin{aligned}
\mathcal{S}= & \int d^{3} x d \tau \psi^{\dagger}\left(\alpha \partial_{\tau}+\mathcal{H}_{0}(-i \nabla)+i e \varphi+g M \phi\right) \psi \\
& +\int d^{3} x d \tau \frac{1}{2}\left[(\nabla \varphi)^{2}+(\nabla \phi)^{2}+\left(\partial_{\tau} \phi\right)^{2}+r \phi^{2}\right],
\end{aligned}
$$

where the momentum cutoff $(\Lambda)$ is assumed and where the Hamiltonian density is $\mathcal{H}_{0}(\mathbf{k})=c_{0} \mathbf{k}^{2}+\sum_{a=1}^{5} \hat{c}_{a} d_{a}(\mathbf{k}) \Gamma_{a}$. Higher-order terms omitted in Eq. (2) prove irrelevant at the QCP. The $d_{a}$ 's (given in Appendix A 1) make a complete basis of the allowed terms quadratic in $k_{j}$, chosen such that $d_{1,2,3}$ belong to a three-dimensional representation (often called $T_{2 g}$ ) and $d_{4,5}$ make a two-dimensional one (commonly referred to as $E_{g}$ ), the $\Gamma_{a}$ 's are anticommuting unit matrices, $\left\{\Gamma_{a}, \Gamma_{b}\right\}=2 \delta_{a b}, \quad \Gamma_{a b}=(-i / 2)\left[\Gamma_{a}, \Gamma_{b}\right]$, $\hat{c}_{1}=\hat{c}_{2}=\hat{c}_{3}=c_{1}$, and $\hat{c}_{4}=\hat{c}_{5}=c_{2}$ (as they should since they belong to the same representation), and symmetry dictates (see Appendices A 2 and A 3) that the order parameter couples via the matrix $M=\Gamma_{45}\left(\in A_{2 g}\right) . e$ is the magnitude of the electron charge, and $g \in \mathbb{R}$ parametrizes the coupling strength of the fermions to the order parameter. As discussed in Appendix A 2, $c_{0,1,2}$ may always be chosen to be positive, without loss of generality. $c_{0}$ parametrizes "particle-hole asymmetry," with $c_{0}=0$ denoting a symmetric band structure. Also, when $c_{0} \leq c_{1} / \sqrt{6}$, in the vicinity of the Gamma point, the bands touch at and only at the Gamma point. We assume that the system parameters fall within this range and find that this is consistent, even when taking into account their nontrivial scale-dependent renormalization by interactions.

The model in Eq. (2) has two phases (see Fig. 1). For $r>r_{c} \sim g^{2}$ (where $r_{c}$ is thereby defined), $\phi$ fluctuates around zero and can be integrated out. This is a magnetically disordered state, and the bare dispersion is quadratic [see Fig. 2(c)]. The resulting model with Coulomb interactions alone describes a non-Fermi liquid phase, as first discussed by Abrikosov and Beneslavskii [22,23] and thoroughly revisited recently [10]. Notably, in this regime, nontrivial scaling exponents arise and the low-energy electronic dispersion renormalizes to become isotropic, i.e., effectively $c_{1} \rightarrow c_{2}$ and $c_{0} \ll c_{1}$. This state is a non-Fermi liquid with neither electronic nor magnetic quasiparticles well defined. For $r<r_{c}$, the expectation value $\langle\phi\rangle \neq 0$, and replacing $\phi \rightarrow\langle\phi\rangle$ causes the twofold degenerate bands to split [see Fig. 2(a)], removing the quadratic touching at $\mathbf{k}=$ 0 in favor of eight linearly dispersing "Weyl points" along the $\langle 111\rangle$ directions: a Weyl semimetal. Weyl semimetals have many unusual properties that have been explored theoretically (see, e.g., Ref. [24]).

\section{RENORMALIZATION GROUP}

\section{A. $1 / N$ expansion}

We now turn to the critical regime. To proceed, we introduce as a formal device $N$ copies of the four fermion fields, replacing $g \rightarrow g / \sqrt{N}$ (resp. ie $\rightarrow i e / \sqrt{N}$ ) and $\Gamma_{a} \rightarrow$ $\Gamma_{a} \otimes 1_{N}\left(1_{N}\right.$ is the $N \times N$ identity matrix). We organize perturbation theory in powers of $1 / N$ and formally work to leading nontrivial order in $1 / N$ for all physical quantities. (a)

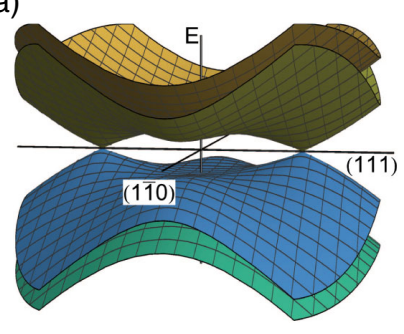

$\langle\phi\rangle \neq 0$ (b)

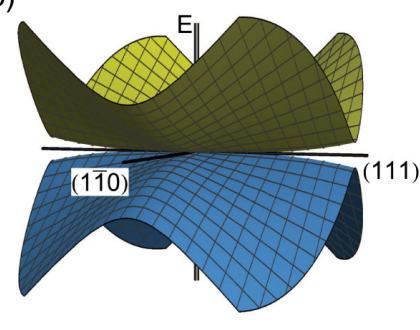

$\langle\phi\rangle=0, c_{1} / c_{2}=e^{-v_{0} \sqrt{\ln k_{0} / k}}$ (c)

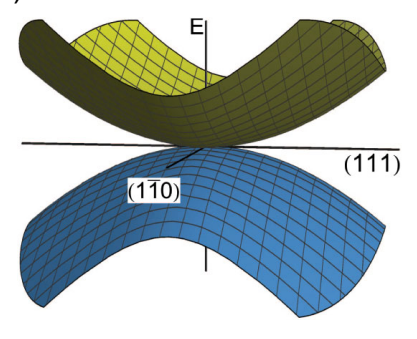

$\langle\phi\rangle=0$

FIG. 2. Two-dimensional cut of the electronic band structure around the Gamma point (a) in the Weyl semimetal, (b) at the quantum critical point, and (c) in the quadratic band touching (Luttinger-Abrikosov-Beneslavskii) phase. In (b), we have included the effect of the irrelevant $c_{1}$ term which produces some weak dispersion along the $\langle 111\rangle$ directions. For more details, see the text at the end of Sec. III D. 
The structure of the renormalization, however, suggests that the results may be independent of $N$ at low energy-see Sec. V B. We require the two boson self-energies in Fig. 3 and, using the dressed boson propagators including this correction, the fermion self-energy and vertex functions in Fig. 4. These diagrams allow a full calculation of the $O(1 / N)$ terms of all critical exponents.

\section{B. Extreme anisotropy limit}

The evaluation of the diagrams is complicated by the three mass parameters of the free fermion propagators. Fortunately, a simplification is possible thanks to the structure of the RG. While the (inverse) mass terms $c_{0}$, $c_{1}$, and $c_{2}$ all have identical engineering dimensions, they, in general, renormalize differently from loop corrections; thus, their ratios flow in the full RG treatment. We find below that, in the critical regime, $c_{0} / c_{2}, c_{1} / c_{2} \rightarrow 0$ under renormalization (arguments as to why this is the only reasonable choice are given in Appendix C 3). This allows technical simplifications in the loop integrals and also has physical consequences that we explore later.

Physically, this limit corresponds to extreme cubic anisotropy. In particular, as $c_{1} / c_{2} \rightarrow 0$, the interband splitting vanishes along the $\langle 111\rangle$ directions, leading to an extended singularity of the electron Green's function. Obviously, the extended singularity is avoided by never actually taking $c_{1}$ all the way to zero. However, as $c_{1}$ becomes small under renormalization, the loop integrals become increasingly dominated by the region around the incipient singularity, which we take advantage of.

\section{Boson self-energy and $N=\infty$ limit}

At $N=\infty$, we need the leading term in the boson selfenergies only. In the loop integrals determining them, the extended singularity of the extreme anistropy limit produces a divergent contribution at nonzero $\mathbf{k}$. Technically, with the assumptions $c_{0} / c_{2}, c_{1} / c_{2} \ll 1$ and $c_{0} / c_{1}<1 / \sqrt{6}$ (shown to be self-consistent below), the low-energy behavior (small $\omega_{n}, \mathbf{k}$ ) may be extracted as (see Appendix B)

$$
\Sigma_{b}\left(\omega_{n}, \mathbf{k}\right)=-r_{b}^{c}+\frac{g_{b}^{2}}{\alpha}\left(\left|\ln c_{1} / c_{2}\right||\mathbf{k}| f_{b}(\hat{\mathbf{k}})+\sqrt{\left|\omega_{n}\right|} C_{b}\right),
$$

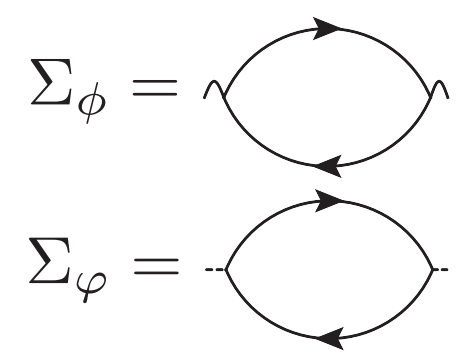

FIG. 3. Boson self-energies for the order parameter $\left(\Sigma_{\phi}\right)$ and electrostatic $\left(\Sigma_{\varphi}\right)$ fields.

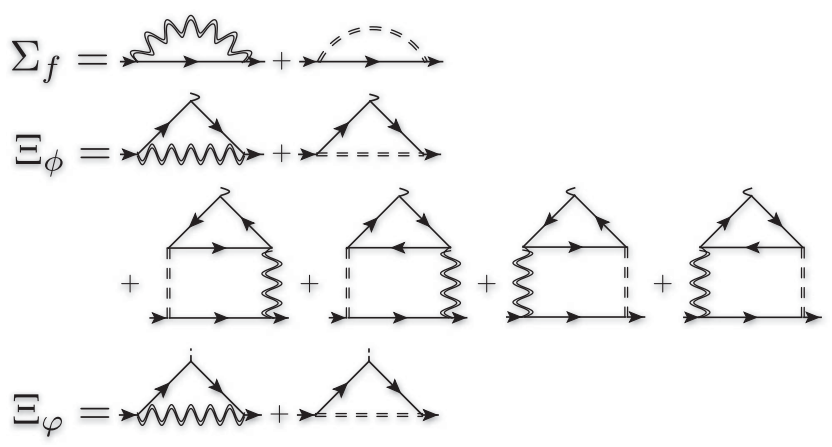

FIG. 4. $1 / N$ diagrams for the fermion self-energy $\Sigma_{f}$ and vertex corrections $\Xi_{\phi}$ and $\Xi_{\varphi}$ (only two-loop diagrams that need to be calculated, i.e., that do not vanish or cancel one another, are shown). Double lines indicate the renormalized boson propagators including the self-energies from Fig. 3. Expressions for these diagrams are given in Appendix $\mathrm{C} 1$.

with $r_{\phi}^{c}=r_{c} \sim g^{2} \Lambda$, where $\Lambda$ is an upper momentum cutoff, $g_{\phi}=g, g_{\varphi}=i e$, and $r_{\varphi}^{c}=C_{\varphi}=0$ follows from charge conservation. $C_{\phi} \approx 1.33$, and the functions $f_{b}(\hat{\mathbf{k}})$ are given as integrals in Appendix B 1.

Note that, at low energy, the dispersive terms in Eq. (3) are much larger than the bare $\mathbf{k}^{2}, \omega_{n}^{2}$ terms that they correct; hence, they dominate the renormalized Green's functions. Thus, in the fermion self-energy and vertex correction, the renormalized boson propagator, $\mathcal{G}_{b}^{-1}=\mathcal{G}_{b ; 0}^{-1}+\Sigma_{b} \approx \Sigma_{b}+$ $r_{b}$ (note $r_{\varphi}=0$ ), must be used. This renormalized boson propagator corresponds to the $N=\infty$ result and already reveals some dramatic features. First, the bosons immediately receive a large anomalous scaling dimension, equal to 1 , and their dynamics becomes damping dominated, with dynamical critical exponents close to 2 . Second, since the damping terms which dominate $\mathcal{G}_{b}^{-1}$ are proportional to $g_{b}^{2}$, it implies that the fermion self-energies, which involve two interaction vertices (see Fig. 4), become $g_{b}$ independent; this is a sign of universality at the QCP.

\section{Fermion self-energy and vertex corrections: RG flows at $1 / N$}

To confirm the assumed scaling of $c_{1} / c_{2}$ and $c_{0} / c_{2}$, and fully determine the critical behavior, we turn to the renormalization group approach. There, as usual, we apply the following rescaling (applicable in real space),

$$
\begin{aligned}
x & \rightarrow e^{\ell} x, \quad \tau \rightarrow e^{\int_{0}^{\ell} d \ell^{\prime} z\left(\ell^{\prime}\right)} \tau, \quad \psi \rightarrow e^{-\int_{0}^{\ell} d \ell^{\prime} \Delta_{\psi}\left(\ell^{\prime}\right)} \psi, \\
\phi & \rightarrow e^{-\int_{0}^{\ell} d \ell^{\prime} \Delta_{\phi}\left(\ell^{\prime}\right)} \phi, \quad \varphi \rightarrow e^{-\int_{0}^{\ell} d \ell^{\prime} \Delta_{\varphi}\left(\ell^{\prime}\right)} \varphi,
\end{aligned}
$$

where $\ell \geq 0$ parametrizes the RG flow. The exponents are left allowed to be scale dependent, as is necessary [25], as we shall see below.

We evaluate the contributions to the fermion propagator and coupling constants due to a small change in the cutoff (which corresponds physically to integrating out modes to 
keep the rescaled cutoff unchanged). Hence, the RG flow equations are obtained by (i) logarithmically differentiating the fermion self-energy and vertex functions with respect to the cutoff $\Lambda$ [which is made soft through a rapidly decaying function $|\mathbf{q}| / \Lambda \mapsto \mathcal{F}(|\mathbf{q}| / \Lambda)][25,26]$ and (ii) identifying the appropriate coefficients of the Taylor expansion (in $k_{i}$ and $\omega_{n}$ ) of the result [27].

We leave most details to Appendix $\mathrm{C}$ and only give one example here. To extract the correction to the mass coefficient $c_{1}$, we first expand the fermion self-energy as

$$
\Sigma_{f}\left(\omega_{n}, \mathbf{k}\right)=\Sigma_{f}^{0} I+\sum_{a=1}^{5} \Sigma_{f}^{a} \Gamma_{a}
$$

( $I$ is the identity matrix) and examine the $\Sigma_{f}^{1}$ component. The RG equation is then

$$
\frac{\partial_{\ell} c_{1}}{c_{1}}=z+1-2 \Delta_{\psi}+\left.\frac{\sqrt{2}}{c_{1}}\left(\partial_{k_{x}, k_{y}}^{2}\left[\Lambda \frac{d}{d \Lambda} \Sigma_{f}^{1}\right]\right)\right|_{\omega_{n}=0, \mathbf{k}=\mathbf{0}}
$$

[we define $d_{1}(\mathbf{k})=k_{x} k_{y} / \sqrt{2}$; see Appendix A 1]. Similar expressions are obtained for renormalizations of the other parameters of the theory: $c_{0}, c_{2}, \alpha, g$, and $i e$. Therefore, for the six equations thereby obtained, there are four unknowns $\left(z, \Delta_{\psi}, \Delta_{\phi}\right.$, and $\left.\Delta_{\varphi}\right)$ that can be chosen to keep four parameters fixed, leaving two left to flow. Here, we find it is possible to keep $\alpha, g$, (ie), and $c_{2}$ fixed, and thus $c_{1} / c_{2}$ and $c_{0} / c_{2}$ will flow. It is this flow that leads to the extreme anisotropy limit noted above. Note that, in doing so, we obtain a critical theory with nonzero coupling of fermions both to order-parameter and Coulomb-potential fluctuations: Both effects are crucial and important in stabilizing the QCP.

To obtain explicit equations, we once again take advantage of the flow to extreme anisotropy. We find that the renormalizations of the couplings depend on $c_{1}$ and $c_{2}$ through $1 /\left(N\left|\ln c_{1} / c_{2}\right|\right)$ or $1 /\left(N\left|\ln c_{1} / c_{2}\right|^{2}\right)$ (expressions are expanded in small $1 /\left|\ln c_{1} / c_{2}\right|$; see Appendix A 2). Finally, we find

$$
\begin{aligned}
z(\ell) & =2-\delta z(\ell), \quad \Delta_{\psi}(\ell)=\frac{3+\eta_{\psi}(\ell)}{2}, \\
\Delta_{b}(\ell) & =\frac{3+\eta_{b}(\ell)}{2},
\end{aligned}
$$

where $\delta z=\frac{0.0634}{\left|\ln c_{1} / c_{2}\right| N}, \eta_{\psi}=\frac{0.287}{\left|\ln c_{1} / c_{2}\right|^{2} N}, \eta_{\phi}=1+\frac{0.510}{\left|\ln c_{1} / c_{2}\right| N}$, and $\eta_{\varphi}=1-\frac{0.127}{\left|\ln c_{1} / c_{2}\right| N}$.

The flow equations may be solved thanks to the equation for $c_{1} / c_{2}$, which is an analytically soluble differential equation involving only $c_{1} / c_{2}$ (see Appendix A 5). Ultimately, we find

$$
\begin{aligned}
\left(c_{1} / c_{2}\right)(\ell) & =e^{-\left(v_{0} / \sqrt{N}\right) \sqrt{\ell+\ell_{0}}} \\
\text { and } \quad\left(c_{0} / c_{1}\right)(\ell) & =\Upsilon_{0} e^{-\left(v_{0}^{\prime} / \sqrt{N}\right) \sqrt{\ell+\ell_{0}}}
\end{aligned}
$$

with $v_{0}=0.202$ and $v_{0}^{\prime}=0.424$ and where $\ell_{0}$ and $\Upsilon_{0}$ are constants that depend on the system's parameters, namely, on $c_{0,1,2}(\ell=0)$. Formally, therefore, both the $c_{0}$ and $c_{1}$ mass terms are irrelevant in the RG sense, but they can be "dangerously irrelevant" insofar as they control certain physical properties (see below). Note also that not only is $c_{0}$ irrelevant, but it also flows to zero faster than $c_{1}$ so that $c_{0} / c_{1}$ becomes small at the QCP; i.e., at long scales, we have the hierarchy $c_{0} \ll c_{1} \ll c_{2}$.

In Fig. 2(b), we have schematically drawn the fermion dispersion corresponding to the behavior in Eq. (8). To do so, the fermion propagator, the peak of which defines the dispersion, is estimated for momentum $\mathbf{k}$ by integrating the RG flow until the rescaled momentum is of order the cutoff, $k e^{\ell}=k_{0}$. Then, using the free fermion propagator at that $k$, with the $c_{1}$ in Eq. (8) at that value of $\ell$, we obtain $c_{1} / c_{2} \sim e^{-v_{0} \sqrt{\ln k_{0} / k}}$. This gives a dispersion that is characteristically flattened along the $\langle 111\rangle$ directions, as shown in Fig. 2(b). We note that in both Figs. 2(b) and 2(c), quasiparticles are in fact not sharp, but nevertheless one can still associate a dispersion with the peak of the spectral function.

Intuition for the irrelevance of $c_{1}$ comes from considering the fermion self-energy $\Sigma_{f}$, which yields the corrections to $c_{0,1,2}$ and to $\alpha$ and is given schematically by $\Sigma_{f}=\mathcal{G}_{\phi} M G_{0} M+\mathcal{G}_{\varphi} G_{0}$ (the contributions from each boson field just add up). In the first term, which represents the dressing of electrons by magnetic fluctuations, the appearance of $M$, which commutes with $\Gamma_{1,2,3}$ but anticommutes with $\Gamma_{4,5}$, portends "opposite" consequences for $c_{1}$ and $c_{2}$. The second term, because of Coulomb effects, tends instead to affect $c_{1}$ and $c_{2}$ identically. Our calculation shows that the former tendency prevails, and $c_{1} / c_{2} \rightarrow 0$ under RG, as claimed above. Conversely, the fact that $c_{0} / c_{1} \rightarrow_{\ell \rightarrow \infty} 0$ should be attributed to the effect of Coulomb forces, which suppress particle-hole asymmetry. Indeed, we have checked that if, in the calculations, we artificially turn off the long-range Coulomb potential, i.e., take $e=0$, the $\mathrm{QCP}$ is unstable and there is no direct, continuous quantum phase transition from the $\mathrm{LAB}$ state [10] to the AIAO one (see Appendix C 5).

\section{PHYSICAL IMPLICATIONS}

\section{A. Scaling properties}

Equations (7) and (8) determine the properties at the QCP. The flows are stable, signifying a continuous QCP. We now turn to a discussion of the physical consequences. First, we consider some scaling properties. For the correlation length $\xi$, we need the flow equation for $\delta r=r-r_{c}$, the deviation from the critical point: $\partial_{\ell}(\delta r)=\nu^{-1}(\delta r)$, 
with $\nu=1 /\left[2-\eta_{\phi}(\ell)-\delta z(\ell)\right]$. This implies, in the usual way, that the correlation length behaves as $\xi \sim(\delta r)^{-\nu}$, up to logarithmic corrections. Also interesting is the orderparameter growth in the AIAO phase. By scaling, $\langle\phi\rangle \sim \xi^{-\Delta_{\phi}} \sim|\delta r|^{\beta}$, with $\beta=\Delta_{\phi} \nu$. Up to logarithmic corrections, this is easily verified by direct calculation in the ordered phase. We also expect the critical temperature of the magnetic state to obey $T_{c} \sim \xi^{-z} \sim|\delta r|^{z \nu}$. In asymptopia, i.e., $\ell \rightarrow \infty$, all the $N$-dependent corrections vanish, and the exponents correspond to those of a saddle-point treatment of $\varphi, \phi$. These are still distinct from the usual orderparameter mean-field theory, as witnessed by the large $\left(\eta_{\phi}^{\infty}=\eta_{\varphi}^{\infty}=1\right)$ anomalous dimensions in this limit, and the unconventional values $\nu^{\infty}=1$ and $\beta^{\infty}=(z \nu)^{\infty}=2$. The latter is noteworthy insofar as it implies an unusually wide critical fan at $T>0$ which is controlled by the QCP (see Fig. 1).

\section{B. Logarithmic corrections and extreme anisotropy}

The RG treatment goes beyond the saddle point in giving the corrections due to finite $c_{1} / c_{2}$, which are small only logarithmically and thus may be significant for physically realistic situations. For example, we find $\langle\phi\rangle \sim$ $(\delta r)^{2} \exp \left[(13.9 / \sqrt{N}) \sqrt{\ln \left(\delta r / r_{0}\right)}\right]$ (see Appendix D 1), where $r_{0}$ is a constant.

The irrelevance of $c_{0}$ and $c_{1}$ has other, more direct, physical consequences. Because of the irrelevance of the former, the low-energy electronic spectrum becomes approximately particle-hole symmetric. The irrelevance of $c_{1}$ has more implications. Obviously, the electronic spectrum develops pronounced cubic anisotropy, with anomalously low-energy excitations along the cubic $\langle 111\rangle$ directions in momentum space. This is in stark contrast to most critical points (for example, of GinzburgLandau type or involving Dirac fermions), which typically have emergent spatial isotropy and even conformal symmetry and Lorentz invariance at the fixed point. These low-energy excitations manifest, for example, in the specific heat $c_{v}$. Since at the Gaussian level the coefficient of $T^{3 / 2}$ diverges as $c_{1}^{-3 / 2}$, we estimate, by using $\ell \sim \frac{1}{z} \ln T_{0} / T$ as a cutoff ( $T_{0}$ is a microscopic energy scale), $c_{v} \sim \exp \left[3 v_{0} /(2 \sqrt{N} \sqrt{z}) \sqrt{\ln \left(T_{0} / T\right)}\right] T^{3 / 2}$, with $z \approx 2$ (see Appendix D 2). We may also obtain many other physical quantities such as the electronic contribution to the magnetic susceptibility. However, we refrain from presenting more of such results here, as they are obtained by straightforward application of the formalism already presented (see, especially, Appendix D). Exceptions to this rule are the electrical resistivity and other transport quantities, which are much more subtle and involve many potential crossovers resulting from the interplay of scattering by order-parameter fluctuations, Coulomb forces, disorder, and umklapp effects, and by the dependence of all of these on directionality in momentum space. This is a formidable problem on which we feel it is inappropriate to provide speculative and possibly incorrect results. We plan to address this problem instead in some detail in a future study.

As mentioned in the Introduction, the emergent anisotropy may also manifest in increasingly "spiky" Fermi surfaces in lightly doped samples near the QCP (see Fig. 1). We estimate the anisotropy of the Fermi surface by standard scaling arguments. For a small but nonzero Fermi energy (chemical potential) $\epsilon_{F}$, which introduces light doping and a small Fermi surface, the scale-dependent renormalizations of the quantum critical regime apply until the Fermi momentum is comparable to the reduced cutoff. Setting the single-particle energy equal to $\epsilon_{F}$ implies that the large Fermi momentum (along the $\langle 111\rangle$ directions) is proportional to $k_{F}^{\text {large }} \sim \sqrt{\epsilon_{F} / c_{1}}$, while the small Fermi momentum (along $\langle 100\rangle$ ) is $k_{F}^{\text {small }} \sim \sqrt{\epsilon_{F} / c_{2}}$. Using the scale-dependent ratio of $c_{1} / c_{2}$ from Eq. (8) and requiring that $k_{F}^{\text {large }}\left(\ell_{c}\right) e^{\ell_{c}} \sim \Lambda$, which is the matching condition determining the $\ell_{c}$ at which the metallic state is reached, we obtain appendi $\ell_{c}$, and hence, finally, with some algebra,

$$
\frac{k_{F}^{\text {large }}}{k_{F}^{\text {small }}} \sim e^{\frac{v_{0}}{2 \sqrt{N}} \ln ^{1 / 2} \sqrt{\epsilon_{F}^{0} / \epsilon_{F}}},
$$

where $\epsilon_{F}^{0} \sim c_{2} \Lambda^{2}$ is a microscopic energy of order the bandwidth.

Although rotational symmetry is strongly broken, the asymptotic vanishing of $c_{1}$ leads to an emergent internal $S O(3)$ symmetry, corresponding to rotating the $\Gamma_{a}$ matrices with $a=1,2,3$ amongst themselves like a vector. The generator of this symmetry is the $S U(2)$ pseudospin I, with

$$
I_{a}=-\frac{1}{4} \epsilon_{a b c} \psi^{\dagger} \Gamma_{b c} \psi=\psi^{\dagger}\left(-\frac{7}{6} J_{a}+\frac{2}{3} J_{a}^{3}\right) \psi,
$$

where $a=x, y, z=1,2,3$. Its integral has $S U(2)$ commutation relations and commutes with the fixed-point Hamiltonian.

\section{DISCUSSION}

\section{A. Comparison to Hertz-Millis and mean-field theories}

In standard Hertz-Millis theory [3,4], the inequality $d+z>4$ implies that the theory is above its critical dimension and thus has mean-field behavior. Although this inequality holds here, taking $z=2$, the conclusion is false. The Hertz-Millis approach assumes the fermions may be innocuously integrated out, and it obtains the inequality above by power counting the $\phi^{4}$ term in the Landau action, which is irrelevant. Instead, here we have strong coupling of fermions with the order parameter, and the coupling term $\sim \phi \psi^{\dagger} \psi$ is marginal, using $z=2, \Delta_{\phi}=2, \Delta_{\psi}=3 / 2$. If one does integrate out the fermions, one obtains a 
nonanalytic $|\phi|^{5 / 2}$ term (see Appendix E), which overwhelms the naïve $\phi^{4}$ one and is again marginal by power counting. This $|\phi|^{5 / 2}$ dependence was obtained previously in Ref. [28], in the context of a mean-field treatment of related transitions. Mean-field-like treatment of transitions in the iridates was also carried out in Refs. [14,29] via Hubbard models. Note, however, that such mean-field analyses integrating out fermions (explicitly or in essence) are not justified and miss important physics.

\section{B. Beyond large $N$}

Our critical theory has some formal similarity to the theory of a two-dimensional nodal nematic QCP in a $d$ wave superconductor [25], insofar as both theories display "infinite anisotropy": In our case, this is due to $c_{1} / c_{2} \rightarrow 0$ under RG. In Ref. [25], it was argued that, at low energies, the critical behavior for the nodal nematic transition becomes universal and independent of $N$. In that problem, one observes this independence already at one-loop order by the fact that, in the perturbative RG expansion, each factor of $1 / N$ is accompanied by a scale-dependent factor that vanishes as $\ell \rightarrow \infty$. In our own calculations, we find the same phenomena: To leading order, each factor of $1 / N$ is accompanied by one or two inverse logarithms of $c_{1} / c_{2}$, so that in fact, the perturbative expansion parameter scales to small values for fixed $N$. Reference [25] takes this observation one step further and verifies that this simplification recurs at higher order, but because of its considerably more complex analytical structure, we have not been able to prove this for the current problem. Nevertheless, the results we have obtained suggest that the critical behavior may in fact be independent of $N$ and hence may apply directly at low energy to the physical case $N=1$. This conclusion is appealing, though we have not shown it rigorously.

\section{Experiments}

With the above results in hand, we comment on the connection to experiments. The theory developed here relies only on cubic symmetry and strong SOC and may therefore apply to a number of materials, provided their bands at the Fermi energy belong to the appropriate irreducible representation. Among those, as emphasized earlier, the family of pyrochlore iridates provides ideal examples: Their $5 d \mathrm{Ir}^{4+}$ electrons are delocalized enough to be conducting, they are strongly coupled, and the symmetries are indeed appropriate. Moreover, the existence of a whole family of compounds provides a natural variable, the rare earth ionic radius, to tune the electronic structure and Hamiltonian parameters. Most importantly, recent $a b$ initio calculations and ARPES experiments have provided very strong evidence for the applicability of the quadratic band touching model of the present paper to $\operatorname{Pr}_{2} \mathrm{Ir}_{2} \mathrm{O}_{7}$. With the other compounds in the $\mathrm{A}_{2} \mathrm{Ir}_{2} \mathrm{O}_{7}$ series exhibiting a magnetic-ordering transition at finite temperatures, and $\mathrm{Pr}_{2} \mathrm{Ir}_{2} \mathrm{O}_{7}$ remaining paramagnetic throughout its phase diagram, the QCP described in this paper might be tuned by alloying the A-site atoms, e.g., $\operatorname{Pr}_{2-x} \mathrm{Y}_{2 x} \mathrm{Ir}_{2} \mathrm{O}_{7}$, or by pressurizing stoichiometric compounds nearby.

Quantum criticality manifests itself in power-law or singular behavior of nearly all physical quantities. For example, all thermodynamic quantities should have powerlaw temperature, pressure, and magnetic-field dependence in the quantum critical regime. This includes heat capacity, susceptibility, Grüneisen parameter, etc. This describes only the electronic contributions to the thermodynamics. Contributions from lattice and localized $f$-moments will also appear in most cases, complicating the comparison with thermodynamics. It is perhaps more promising to consider electrical transport coefficients since only extended electronic states carry current. This includes DC and optical conductivity, Hall effect, etc. However, the conductivity is a rather singular quantity: For the generic model with $c_{0} \neq 0$, the lack of particle-hole symmetry combined with translational invariance implies that the DC conductivity is infinite for $T>0$. The physical conductivity is rendered finite by a delicate combination of subsidiary processes that lead to momentum relaxation, mixing of current and momentum, and other effects. Hence, the conductivity does not readily follow from the theory of the quantum critical point itself without further assumptions. We postpone a discussion of transport phenomena to future work.

Perhaps the most direct observation of strongly coupled quantum criticality is the "incoherent" nature of both the order-parameter and quasiparticle excitations. The quantum critical theory predicts power-law scaling of both the dynamical spin susceptibility (measured by inelastic neutron or x-ray scattering), which describes the fluctuating order parameter modes, and the single particle spectral function (measured in angle-resolved photoemission), which describes the strongly scattered quasiparticles. An explicit calculation of the fermion spectral function measured in angle-resolved photoemission has been made neither here nor for the non-Fermi liquid paramagnetic state [10], and it is an important problem for future theory. However, in general, the weak logarithmic flow of the Hamiltonian parameters signifies large self-energy corrections, and behavior somewhat similar to marginal Fermi-liquid theory may be expected. Both magnetic and electronic spectral functions are promising avenues for future research.

We also mention some possible complications in the iridates. Impurity scattering is a relevant perturbation and hence important at low energy close to the band touching. Therefore, our results will apply best in the cleanest samples. Finally, in many of the pyrochlore iridates, the A-site ion hosts rare-earth moments, which were not included here. They only weakly couple to the Ir electrons and to themselves, so they are only important at low energies. On the antiferromagnetic side of the QCP, the Ir spins act as strong local effective magnetic fields, locking 
the A-site spins. However, when the Ir sites are not ordered, as in $\operatorname{Pr}_{2} \operatorname{Ir}_{2} \mathrm{O}_{7}$, the A-site ions will be important below a few Kelvins. Several authors have proposed scenarios based on RKKY interactions [30-32], but the quantum critical theory expounded here should be an apt starting point for a systematic analysis.

Finally, we address models presented in the literature. Some phenomenological tight-binding models in the literature (e.g., Ref. [29]) suggest that the valence or conduction bands may bend and accidentally cross the Fermi energy away from the zone center, thereby shifting the Fermi level away from the nodal point (and creating other pockets elsewhere in the zone). If such an accidental crossing of the Fermi energy were to occur, our results would hold for energies and/or temperatures above this shift energy, but a crossover would occur below it. We emphasize that this is nongeneric: The quadratic band touching is both the minimal situation and a generic one, and it is completely stable to local perturbations. Moreover, the large spatial extent of the $5 d$ Ir orbitals and complexities of crystal fields makes the reliability of ad hoc tight-binding models questionable. As mentioned earlier, recent $a b$ initio calculations [11] and angle-resolved photoemission experiments [12] on $\operatorname{Pr}_{2} \mathrm{Ir}_{2} \mathrm{O}_{7}$ both show no such accidental band crossing, which strongly supports the applicability to this material of the quadratic band touching model studied here.

\section{ACKNOWLEDGMENTS}

We thank Cenke Xu and Yong-Baek Kim for discussions on prior work and Max Metlitski for pointing out Ref. [25] to us, and we acknowledge Ru Chen, Satoru Nakatsuji, and Takeshi Kondo for sharing unpublished data. The numerical integrals were performed in $\mathrm{C}$ using the Cuba library [33], and the Feynman diagrams in Figs. 3 and 4 were drawn with JaxoDraw [34]. L. S. and L. B. were supported by the DOE through Grant No. DE-FG02-08ER46524, and E.-G. M. was supported by the MRSEC Program of the National Science Foundation under Grant No. DMR 1121053.

\section{APPENDIX A: NOTATIONS AND SYMMETRIES}

In this section, we provide more information about the notations used in the main text and a more detailed discussion of the symmetries at play.

In reciprocal space, the action, Eq. (2) in the main text, is

$$
\begin{aligned}
\mathcal{S}= & \int_{-\infty}^{\infty} \frac{d \omega_{n}}{2 \pi} \int_{\Lambda} \frac{d^{3} k}{(2 \pi)^{3}}\left[\phi_{-\omega_{n},-\mathbf{k}}\left(\frac{\omega_{n}^{2}}{2}+\frac{\mathbf{k}^{2}}{2}+\frac{r}{2}\right) \phi_{\omega_{n}, \mathbf{k}}+\varphi_{-\omega_{n},-\mathbf{k}}\left(\frac{\mathbf{k}^{2}}{2}\right) \varphi_{\omega_{n}, \mathbf{k}}+\psi_{\omega_{n}, \mathbf{k}}^{\dagger}\left(-\alpha i \omega_{n}+c_{0} \mathbf{k}^{2}+\sum_{a=1}^{5} \hat{c}_{a} d_{a}(\mathbf{k}) \Gamma_{a}\right) \psi_{\omega_{n}, \mathbf{k}}\right. \\
& \left.+g \int_{-\infty}^{\infty} \frac{d \omega_{n}^{\prime}}{2 \pi} \int_{\Lambda} \frac{d^{3} k^{\prime}}{(2 \pi)^{3}} \phi_{\omega_{n}^{\prime}-\omega_{n}, \mathbf{k}^{\prime}-\mathbf{k}} \psi_{\omega_{n}^{\prime}, \mathbf{k}^{\prime}}^{\dagger} M \psi_{\omega_{n}, \mathbf{k}}+i e \int_{-\infty}^{\infty} \frac{d \omega_{n}^{\prime}}{2 \pi} \int_{\Lambda} \frac{d^{3} k^{\prime}}{(2 \pi)^{3}} \varphi_{\omega_{n}^{\prime}-\omega_{n}, \mathbf{k}^{\prime}-\mathbf{k}} \psi_{\omega_{n}^{\prime}, \mathbf{k}^{\prime}}^{\dagger} \psi_{\omega_{n}, \mathbf{k}}\right]
\end{aligned}
$$

where all the notations are defined in Appendix A. Throughout the appendices, for ease of presentation, we shift the QCP so that $r_{c}=0$.

\section{Fermion Hamiltonian}

The fermionic Hamiltonian density in the disordered (quadratic band-touching) phase reads

$$
\begin{aligned}
\mathcal{H}_{0}(\mathbf{k}) & =\alpha_{1} \mathbf{k}^{2}+\alpha_{2}(\mathbf{k} \cdot \mathbf{J})^{2}+\alpha_{3}\left(k_{x}^{2} J_{x}^{2}+k_{y}^{2} J_{y}^{2}+k_{z}^{2} J_{z}^{2}\right) \\
& =c_{0} \mathbf{k}^{2}+\sum_{a=1}^{5} \hat{c}_{a} d_{a}(\mathbf{k}) \Gamma_{a},
\end{aligned}
$$

where $\hat{c}_{1}=\hat{c}_{2}=\hat{c}_{3}=c_{1}$ and $\hat{c}_{4}=\hat{c}_{5}=c_{2}$. The first line uses the conventional Luttinger parameters $\left(\alpha_{1,2,3}\right)$ in the $j=3 / 2$ matrix representation [35], and the second line is the form used in the main text. The Gamma matrices $\left(\Gamma_{a}\right)$ form a Clifford algebra, $\left\{\Gamma_{a}, \Gamma_{b}\right\}=2 \delta_{a b}$, and have been introduced as described in the literature [36]. Note that $c_{0}$ quantifies the particle-hole asymmetry, while $\left|c_{1}-c_{2}\right|$ naturally characterizes the cubic anisotropy. The energy eigenvalues are $\mathbf{E}_{ \pm}(\mathbf{k})=c_{0} \mathbf{k}^{2} \pm E(\mathbf{k})$, where $E(\mathbf{k})=$ $\sqrt{\sum_{a=1}^{5} \hat{c}_{a}^{2} d_{a}^{2}(\mathbf{k})}$ and

$$
\begin{array}{ll}
d_{1}(\mathbf{k})=\frac{k_{x} k_{y}}{\sqrt{2}}, & d_{2}(\mathbf{k})=\frac{k_{x} k_{z}}{\sqrt{2}}, \quad d_{3}(\mathbf{k})=\frac{k_{y} k_{z}}{\sqrt{2}} \\
d_{4}(\mathbf{k})=\frac{k_{x}^{2}-k_{y}^{2}}{2 \sqrt{2}}, & d_{5}(\mathbf{k})=\frac{2 k_{z}^{2}-k_{x}^{2}-k_{y}^{2}}{2 \sqrt{6}} .
\end{array}
$$

It is very important to note that, in the limit $c_{0,1} \rightarrow 0, E(\mathbf{k})$ and the energy spectrum $E_{ \pm}(\mathbf{k})$ become gapless along the $\langle 111\rangle$ directions. When needed, a "regularization" is then possible, for example, by introducing higher-momentum dependence in $c_{1,2}$, e.g., $c_{1,2} \rightarrow c_{1,2}+\lambda \mathbf{k}^{2}$.

It is straightforward to relate the coefficients used in the main text to the Luttinger $\alpha_{i}$ parameters. This can be done by expressing the spin operators in terms of the Gamma matrices by using, for example, the equalities 


$$
\begin{aligned}
& J_{x}=\frac{\sqrt{3}}{2} \Gamma_{15}-\frac{1}{2}\left(\Gamma_{23}-\Gamma_{14}\right), \\
& J_{y}=-\frac{\sqrt{3}}{2} \Gamma_{25}+\frac{1}{2}\left(\Gamma_{13}+\Gamma_{24}\right), \\
& J_{z}=-\Gamma_{34}-\frac{1}{2} \Gamma_{12},
\end{aligned}
$$

where $\Gamma_{a b}=\frac{1}{2 i}\left[\Gamma_{a}, \Gamma_{b}\right]$.

The fermion bare Green's function is

$$
G_{\omega_{n}, \mathbf{k}}^{0}=\frac{1}{-i \alpha \omega_{n}+\mathcal{H}_{0}(\mathbf{k})}=\frac{1}{-i \alpha \omega_{n}+\mathrm{E}_{\epsilon}(\mathbf{k})} \mathrm{P}_{\epsilon}(\mathbf{k}),
$$

where the sum over $\epsilon= \pm 1$ is implicit and $\mathrm{P}_{\epsilon}(\mathbf{k})=$ $\frac{1}{2}\left(1+\epsilon\left[\left(\mathcal{H}_{0}(\mathbf{k})-c_{0} \mathbf{k}^{2}\right) / E(\mathbf{k})\right]\right)$ is a projection operator, $\mathrm{P}_{\epsilon}^{2}(\mathbf{k})=1$.

\section{Symmetries}

It is useful to recap the symmetries of the system in the absence of all-in-all-out order and to detail the remaining symmetries in its presence.

As defined above and in Refs. [10,36], the $\Gamma_{a}$ matrices are even under time-reversal and inversion symmetry, while the $\Gamma_{a b}$ are even under inversion but odd under time-reversal symmetry.

As is well known for some semiconductors, like $\mathrm{HgTe}$, the touching of four bands at the Gamma point is protected by cubic symmetries (the bands at the Gamma point belong to a four-dimensional representation of the cubic group $O_{h}$ ), and the absence of a linear term follows from timereversal and cubic (inversion) symmetries. Moreover, thanks to inversion and time-reversal symmetries, all bands are doubly degenerate away from the Gamma point.

The magnetic-order-parameter field $\phi$ transforms as follows under the symmetries of the "disordered" system. It is odd under time-reversal symmetry (since the spins $\vec{S} \rightarrow$ $-\vec{S}$ under time-reversal symmetry), and so only the (timereversal-odd) $\Gamma_{a b}$ can couple to it. It is even under inversion (since $\vec{S} \rightarrow \vec{S}$ under inversion), unchanged under threefold rotations, and odd under the allowed reflections of the pyrochlore lattice. A single Gamma matrix, namely, $\Gamma_{45} \propto$ $J_{x} J_{y} J_{z}+J_{z} J_{y} J_{x}[10,36]$ (see below), transforms identically.

The Hamiltonian at fixed $\mathbf{k}$, i.e., $\mathcal{H}_{0}(\mathbf{k})$, together with the coupling to the order parameter with $\phi \neq 0$, which we call $\mathcal{H}_{1}(\mathbf{k})$, has the following transformation properties. For $\mathbf{k} \|\langle 111\rangle, \mathcal{H}_{1}$ is invariant under threefold rotations about $\mathbf{k}$ and reflections with respect to planes that contain $\mathbf{k}$. For $\mathbf{k}=\mathbf{0}$, there is, in addition, inversion symmetry. The symmetry group at $\mathbf{k}=\mathbf{0}$ then decomposes the four bands of interest into two two-dimensional representations. For $\mathbf{k} \neq \mathbf{0}$, symmetries do not impose bands to cross, hence making any crossings "accidental." However, it is noteworthy that the purely quadratic Hamiltonian $\mathcal{H}_{0}$ that we study, with $c_{0} \leq c_{1} / \sqrt{6}$ and $c_{1} \leq c_{2} / \sqrt{6}$, in the presence of the linear coupling to the order parameter $\phi \psi^{\dagger} \Gamma_{45} \psi$ leads inevitably to band crossings along the $\langle 111\rangle$ directions.

Note that the system in the presence of an external applied magnetic field, discussed in Ref. [10], is less symmetric. The system's Hamiltonian at fixed $\mathbf{k}$, which we call $\mathcal{H}_{2}(\mathbf{k})$, is only invariant under threefold rotations about $\mathbf{k}$ if both the magnetic field and $\mathbf{k}$ point along the same $\langle 111\rangle$ direction. For $\mathbf{k}=\mathbf{0}$, the system has, in addition, inversion symmetry, but all the representations of the symmetry group are one dimensional anyway, and there is a priori no degeneracy at $\mathbf{k}=\mathbf{0}$. Away from $\mathbf{k}=\mathbf{0}$, any band crossing is, again, accidental.

It is important to note that, although no crossings are required by symmetry, once the crossings occur, their properties are "stable" in the sense that (i) no symmetrypreserving perturbation will remove them, (ii) the dispersion along the crossings will remain linear, and (iii) they will not move away from the $\langle 111\rangle$ axes.

By appropriately transforming the Gamma matrices with transformations not belonging to the cubic group, one can show that the signs of $c_{0,1,2}$ may always be taken to be positive. Therefore, throughout the paper, we assume $c_{0,1,2} \geq 0$. We also assume $c_{0} \leq c_{1} / \sqrt{6}$; i.e., we assume the two sets of bands have opposite curvatures in all directions at the Gamma point or, in other words, that the Fermi energy goes through the band-touching point.

\section{Couplings}

The long-range Coulomb interaction is described by introducing the Hubbard-Stratonovich field $\varphi$, which couples to the density of fermions.

The all-in-all-out operator is represented by the timereversal symmetry-breaking Ising field $(\phi)$ corresponding to $J_{x} J_{y} J_{z}+J_{z} J_{y} J_{x}$ in Luttinger's notation [35]. In terms of the Gamma matrices, the order parameter is $\Gamma_{45} \sim$ $J_{x} J_{y} J_{z}+J_{z} J_{y} J_{x}$. Thus, finally, the interaction part of the action is the "vertex term" given, in real space and imaginary time, by

$$
\mathcal{S}_{\text {vertex }}=\int d^{3} x d \tau \psi^{\dagger}\left[i e \varphi+g \phi \Gamma_{45}\right] \psi,
$$

where $\psi$ is the four-component spinor field. Upon extending the field space to $N$ flavors of fermions, this term becomes

$$
\mathcal{S}_{\text {vertex }} \rightarrow \frac{1}{\sqrt{N}} \int d^{3} x d \tau \psi^{\dagger}\left[i e \varphi+g \phi \Gamma_{45}\right] \psi .
$$

\section{Green's function and self-energy conventions}

We use the following conventions for the boson Green's functions, $\mathcal{G}_{b ; \omega_{n}, \mathbf{k}}$ with $b=\phi, \varphi$, fermion Green's function, $G_{\omega_{n}, \mathbf{k}}$, boson self-energies, $\Sigma_{b}\left(\omega_{n}, \mathbf{k}\right)$ and fermion selfenergy, $\Sigma_{f}\left(\omega_{n}, \mathbf{k}\right)$ : 


$$
\begin{aligned}
\mathcal{G}_{\varphi ; \omega_{n}, \mathbf{k}} & =\left\langle\varphi_{-\mathbf{k}} \varphi_{\mathbf{k}}\right\rangle=\frac{1}{\mathbf{k}^{2}+\Sigma_{\varphi}(\mathbf{k})}, \\
\mathcal{G}_{\phi ; \omega_{n}, \mathbf{k}} & =\left\langle\phi_{-\omega_{n},-\mathbf{k}} \phi_{\omega_{n}, \mathbf{k}}\right\rangle=\frac{1}{\mathbf{k}^{2}+\omega_{n}^{2}+r+\Sigma_{\phi}\left(\omega_{n}, \mathbf{k}\right)}, \\
G_{\omega_{n}, \mathbf{k}}^{\mu \nu} & =\left\langle\psi_{\omega_{n}, \mathbf{k}}^{\mu} \psi_{\omega_{n}, \mathbf{k}}^{\nu}\right\rangle \\
& =\left[-i \alpha \omega_{n}+\mathcal{H}_{0}(\mathbf{k})+\Sigma_{f}\left(\omega_{n}, \mathbf{k}\right)\right]^{-1},
\end{aligned}
$$

where $\mu, \nu=1, \ldots, 4$ (or $1, \ldots, 4 N$ ) but they are omitted throughout. The "bare propagators" are denoted with the subscript or superscript " 0. ."

\section{APPENDIX B: ASYMPTOTIC LIMITS OF THE BOSONIC SELF-ENERGIES}

We first evaluate the boson self-energies in the large- $N$ limit. They are given by

$$
\begin{aligned}
& \Sigma_{b}\left(\omega_{n}, \mathbf{k}\right) \\
& \quad=\frac{g_{b}^{2}}{N} \int_{\Lambda} \frac{d^{3} q}{(2 \pi)^{3}} \int_{-\infty}^{+\infty} \frac{d \Omega_{n}}{2 \pi} \operatorname{Tr}\left[G_{\Omega_{n}, \mathbf{q}}^{0} M_{b} G_{\Omega_{n}+\omega_{n}, \mathbf{q}+\mathbf{k}}^{0} M_{b}\right],
\end{aligned}
$$

where $g_{\varphi}=i e, g_{\phi}=g, M_{\varphi}=I$, and $M_{\phi}=\Gamma_{45}$ ( $I$ is the identity matrix). Here, the subscript $\Lambda$ in the $q$ integral indicates that an ultraviolet cutoff is required to keep $\Sigma_{b}(0, \mathbf{0})$ finite. This determines the (nonuniversal) location of the QCP. However, we seek the corrections to this term for nonzero frequency and momenta, which are cutoff independent and will therefore be obtained below without further discussion of $\Lambda$. We will return later to the role of the cutoff when considering fermionic self-energy terms and treat it in more detail. The explicit expression for $\Sigma_{b}\left(\omega_{n}, \mathbf{k}\right)$ at $c_{0} \leq c_{1} / \sqrt{6}$ is

$$
\Sigma_{b}\left(\omega_{n}, \mathbf{k}\right)=\frac{-g_{b}^{2}}{\alpha} \sum_{\epsilon= \pm} \int_{\Lambda} \frac{d^{3} q}{(2 \pi)^{3}}\left[\frac{E_{\mathbf{q}, \mathbf{k}}^{+}+E_{\mathbf{q}, \mathbf{k}}^{-}+\epsilon 2 c_{0} \mathbf{q} \cdot \mathbf{k}}{\alpha^{2} \omega_{n}^{2}+\left(E_{\mathbf{q}, \mathbf{k}}^{+}+E_{\mathbf{q}, \mathbf{k}}^{-}+\epsilon 2 c_{0} \mathbf{q} \cdot \mathbf{k}\right)^{2}}\right]\left(1-\frac{F_{b ; \mathbf{q}, \mathbf{k}}}{E_{\mathbf{q}, \mathbf{k}}^{+} E_{\mathbf{q}, \mathbf{k}}^{-}}\right)
$$

where $\quad E_{\mathbf{q}, \mathbf{k}}^{ \pm}=E(\mathbf{q} \pm \mathbf{k} / 2) \quad$ and $\quad F_{b ; \mathbf{q}, \mathbf{k}}=$ $\sum_{a=1}^{5}\left(\varepsilon_{a}\right)^{b} \hat{c}_{a}^{2} d_{a}^{\mathbf{q}, \mathbf{k}}(\mathbf{q}-\mathbf{k} / 2) d_{a}(\mathbf{q}+\mathbf{k} / 2), \quad$ with $\quad \varepsilon=$ $(111-1-1)$ and $b=0$ (resp. $b=1$ ) for $b=\varphi$ (resp. $b=\phi)$. Note that $\Sigma_{b}$ is $O(1)$ [and not $O(1 / N)$ ]; mathematically, this is because of the trace, which yields a factor of $N$.

As mentioned above, the boson self-energy $\Sigma_{\phi}(0, \mathbf{0})$ is finite but depends upon the cutoff $\left[\Sigma_{\phi}(0, \mathbf{0})\right.$ is proportional to $\Lambda$ ]. Again, this determines the location of the QCP at $N=\infty$, and when we focus on the critical theory, this zero-frequency zero-momentum contribution is exactly canceled by the bare value of $r$. Hence, we are left with the corrections at nonzero frequency and momenta, which we isolate by considering the selfenergy difference $\Sigma_{b}\left(\omega_{n}, \mathbf{k}\right)-\Sigma_{b}(0, \mathbf{0})$ (for $b=\varphi$, the second term is zero by charge conservation). This difference is finite and cutoff independent. In the $c_{0,1} \rightarrow 0$ limit, which will be the case in the critical theory, the self-energy differences show logarithmic divergences, i.e., contain $\left|\ln c_{1} / c_{2}\right|$. Conveniently, as mentioned in the main text, the latter will act as a control parameter [25], in addition to $N$, in the critical theory.

In the following, we thereby obtain the one-loop bosonic self-energy,

$$
\begin{aligned}
& \Sigma_{b}\left(\omega_{n}, \mathbf{k}\right)-\Sigma_{b}(0, \mathbf{0}) \\
& \quad=\frac{g_{b}^{2}}{\alpha}\left(|\mathbf{k}| f_{b}(\hat{\mathbf{k}})\left|\ln c_{1} / c_{2}\right|+\sqrt{\left|\omega_{n}\right|} C_{b}\right) .
\end{aligned}
$$

For future convenience, we henceforth take $c_{2}=1$ and denote $c=c_{1}$. It is straightforward to obtain the coefficients of the frequency dependence, $C_{b}$. Because $\Sigma_{b}$ is larger than the bare term at $r=0$, which goes as $\mathbf{k}^{2}+\omega_{n}^{2}$, throughout this work, we take $\mathcal{G}_{b} \rightarrow \Sigma_{b}^{-1}$, where $\mathcal{G}_{b}$ is a full boson Green's function. Finally, note that we used an expansion in small $1 /\left|\ln c_{1} / c_{2}\right|$ of $\Sigma_{b}^{-1}$, i.e., of the inverse of Eq. (B2), in some of the calculations.

By evaluating $\Sigma_{b}\left(\omega_{n}, \mathbf{0}\right)-\Sigma_{b}(0, \mathbf{0})$, we find $C_{\varphi}=0$ and $C_{\phi}=1.33$ by taking $\alpha=1, c_{1}=0$, and $c_{2}=1$. Note that in the $c_{1} / c_{2} \rightarrow 0$ limit, the frequency dependence is subdominant and the bosonic propagator becomes static.

We now extract the nontrivial logarithmic momentum dependence $f_{b}(\hat{\mathbf{k}})$.

\section{Coefficient of the logarithm}

As mentioned above, when $c_{0,1}=0$, to which the theory flows at the QCP, the energy $E(\mathbf{k})$ and spectrum $\mathrm{E}_{ \pm}(\mathbf{k})$ vanish for any $\mathbf{k} \|\langle 111\rangle$, which renders the self-energy difference, $\Sigma_{b}\left(\omega_{n}, \mathbf{k}\right)-\Sigma_{b}(0, \mathbf{0})$, divergent. The appearance of a divergence is subtle: For general $\mathbf{k}$, the denominator in Eq. (B2) appears relatively well behaved since the singularity occurs only when both $\mathbf{q}+\mathbf{k} / 2$ and $\mathbf{q}-\mathbf{k} / 2$ lie along a $\langle 111\rangle$ axis. The singularity actually arises from the regions of integration at large $|\mathbf{q}|$ along these directions, where $|\mathbf{k}| \ll|\mathbf{q}|$, so that both energies are small. We analyze it below. In the limit $0 \leq c_{0} \ll c_{1} \ll c_{2}=1$ (i.e., with $c_{1}$ nonzero and small), which is the actual behavior in the RG flows, the divergence is removed, and the result is large in $\left|\ln c_{1} / c_{2}\right|$. In this subsection, we extract the leading result in this limit. Notably, in this limit, the result is independent of $c_{0}$ and can be approximated by simply taking $c_{0}=0$. 
To extract the coefficient of the logarithm, $f_{b}(\hat{\mathbf{k}})$, we rotate to bases whose $x$ axes point along one of the $\langle 111\rangle$ directions and make a change of variables such that

$$
\left\{\begin{array}{l}
\hat{\mathbf{e}}_{1}=\left(s_{1}, s_{2}, s_{3}\right) / \sqrt{3} \\
\hat{\mathbf{e}}_{2}=\left(0, s_{2},-s_{3}\right) / \sqrt{2} \\
\hat{\mathbf{e}}_{3}=\left(-2 s_{1}, s_{2}, s_{3}\right) / \sqrt{6}
\end{array} \text { and } \quad \mathbf{q}=\frac{Q}{c_{1}} \hat{\mathbf{e}}_{1}+u \hat{\mathbf{e}}_{2}+v \hat{\mathbf{e}}_{3},\right.
$$

where $s_{i}= \pm 1$ (allows to span the eight $\langle 111\rangle$ directions). This rewriting is chosen so that for $Q, u, v$ of $O(1)$, the region near the $\left(s_{1} s_{2} s_{3}\right)$ ray is singled out. The Jacobian of this coordinate transformation is $\mathcal{J}_{0}=\left|s_{1} s_{2} s_{3} / c_{1}\right|$. Now, we rewrite the functions involved in the integrand of the self-energies, Eq. (B2), in these new coordinates, and we obtain the leading asymptotic behavior of each such function at small $c_{1}$.

For example, we find

$$
E_{\mathbf{q}, \mathbf{k}}^{ \pm} \approx \frac{1}{c_{1}} \epsilon_{Q, u, v ; k_{1}, k_{2}, k_{3}}^{ \pm} \text {and } F_{b ; \mathbf{q}, \mathbf{k}} \approx \frac{1}{c_{1}^{2}} \gamma_{Q, u, v ; k_{1}, k_{2}, k_{3}}^{b},
$$

where the $\epsilon^{ \pm}$and $\gamma^{b} \quad(b=\varphi, \phi)$ are functions of $\left\{Q, u, v, k_{1}, k_{2}, k_{3}\right\}$ (and, of course, of the $s_{i}$ 's) only. We are then in a position to take the logarithmic derivatives of the boson self-energies. A major simplification thereby occurs: The frequency dependence drops out of $\Sigma_{b}\left(\omega_{n}, \mathbf{k}\right)-\Sigma_{b}\left(\omega_{n}, \mathbf{0}\right)$. We find

$$
\begin{aligned}
& \frac{\alpha}{g_{b}^{2}} c_{1} \partial_{c_{1}}\left[\Sigma_{b}\left(\omega_{n}, \mathbf{k}\right)-\Sigma_{b}\left(\omega_{n}, \mathbf{0}\right)\right] \\
& \quad=\sum_{s_{1}, s_{2}, s_{3}= \pm 1} \int_{0}^{+\infty} \frac{d Q}{2 \pi} \int_{-\infty}^{+\infty} \frac{d u}{2 \pi} \int_{-\infty}^{+\infty} \frac{d v}{2 \pi} \mathcal{K}_{s_{1} s_{2} s_{3}}^{b} \\
& =f_{b}(\mathbf{k})=|\mathbf{k}| f_{b}(\hat{\mathbf{k}})
\end{aligned}
$$

where

$$
\begin{aligned}
\mathcal{K}_{s_{1}, s_{2}, s_{3}}^{b}= & 9 \sqrt{2} Q\left[\frac{3\left(a_{0}-h_{0}^{b}\right)}{a_{0}^{5 / 2}}\right. \\
& \left.+\frac{2\left(h^{b}\left(a_{+}+\sqrt{a_{+}} \sqrt{a_{-}}+a_{-}\right)-3 a_{+} a_{-}\right)}{a_{-}^{2} a_{+}^{3 / 2}+a_{+}^{2} a_{-}^{3 / 2}}\right] .
\end{aligned}
$$

In the above formula, we introduced several expressions:

$$
\begin{gathered}
\kappa=s_{1} s_{2} k_{x} k_{y}+s_{1} s_{3} k_{x} k_{z}+s_{2} s_{3} k_{y} k_{z}, \\
h_{0}^{\phi}=3\left[Q^{2}-2\left(u^{2}+v^{2}\right)\right] \\
a_{0}=h_{0}^{\varphi}=3\left[Q^{2}+2\left(u^{2}+v^{2}\right)\right], \\
h^{\phi}=h_{0}^{\phi}+\left(\mathbf{k}^{2}-\kappa\right),
\end{gathered}
$$

$$
\begin{gathered}
h^{\varphi}=h_{0}^{\varphi}-\left(\mathbf{k}^{2}-\kappa\right), \\
a_{ \pm}=a_{0}+\left(\mathbf{k}^{2}-\kappa \pm 3 \sqrt{2} u\left(s_{2} k_{y}-s_{3} k_{z}\right)\right. \\
\left. \pm \sqrt{6} v\left(s_{2} k_{y}+s_{3} k_{z}-2 s_{1} k_{x}\right)\right),
\end{gathered}
$$

where all the functions defined above, namely, $h_{0}^{b}, a_{0}, h^{b}$, $a_{ \pm}$, and $\mathcal{K}^{b}(b=\phi, \varphi)$, are taken at $\left\{Q, u, v, k_{x}, k_{y}, k_{z}\right\}$ (and are also functions of the $s_{i}$ 's, although we have written the latter explicitly for $\mathcal{K}^{b}$ only). Note that the integrations over $u$ and $v$ are taken all the way from $-\infty$ to $+\infty$, although the sum over the eight directions, $\sum_{s_{1}, s_{2}, s_{3}}$, is also taken. This is because, for nonzero $c_{1}$, the $u, v$ integrations have a priori upper bounds of order $Q / c_{1}$, which is taken to infinity. In the present order of limits, all contributions arise from regions of angular width of order $c_{1}$ from the $\langle 111\rangle$ rays.

The integrals, Eq. (B6), are evaluated thanks to the Cuba library, using the "Cuhre" routine [33].

\section{Approximation}

Since $f_{b}$ is very smooth (see Fig. 5), we approximate it by a low-order polynomial of $\mathbf{k}$ in order to be able to take accurate derivatives of $f_{b}$ as required to compute the flow of $c_{1}$ (and $c_{2}$ )-see Appendix C. Imposing cubic symmetry, the most general polynomial to order 6 can take the form

$$
\frac{1}{f_{b}(\hat{\mathbf{k}})} \approx m_{1}^{b}+m_{2}^{b}\left(\hat{k}_{x}^{4}+\hat{k}_{y}^{4}+\hat{k}_{z}^{4}\right)+m_{3}^{b} \hat{k}_{x}^{2} \hat{k}_{y}^{2} \hat{k}_{z}^{2}
$$

and fits with $m_{1}^{\phi}=2.356, m_{2}^{\phi}=-0.130$, and $m_{3}^{\phi}=4.136$, and with $m_{1}^{\varphi}=-4.704, m_{2}^{\varphi}=0.264$, and $m_{3}^{\varphi}=-8.253$ provide excellent approximations: The square roots of the means of the squares are $R_{\phi}=0.0049$ and $R_{\varphi}=0.0049$,

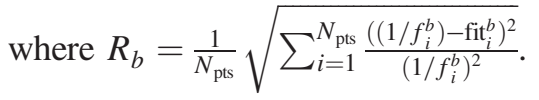

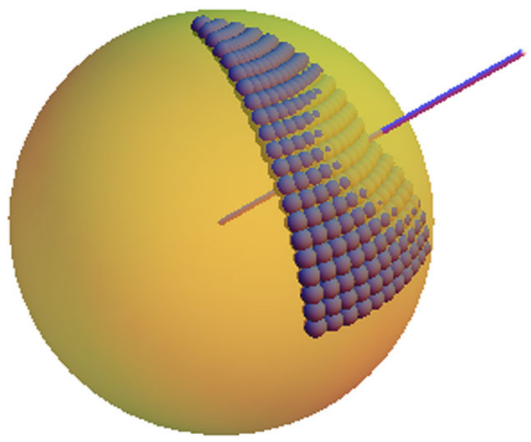

FIG. 5. Plot of $f_{\phi}(\hat{\mathbf{k}}) / f_{\phi}(001)$. The line represents a $\langle 111\rangle$ direction. The whole surface can be obtained from the plotted points by applying cubic symmetries (note that the set of plotted points is larger than the minimal set of points). The yellow surface is a sphere of radius $f_{\phi}(001)$. 


\section{APPENDIX C: RG EQUATIONS}

As discussed in the main text, 24 Feynman diagrams are necessary to determine the RG equations: Two boson selfenergies $\Sigma_{b}$, given in Appendix $\mathrm{B}$, two fermion selfenergies $\Sigma_{f ; b}$, and 20 vertex corrections [the one-loop $\Xi_{b ;(1) ; b^{\prime}}$ (four) and the two-loop $\Xi_{b ;(2) ; b^{\prime}, b^{\prime \prime}, \eta}$ (16-12 of which either vanish identically or cancel each other out), with $b, b^{\prime}, b^{\prime \prime}=\varphi, \phi$ and $\eta= \pm 1$. The notation is expected to be transparent, and the expressions can be read off in Eqs. (C1)-(C3). We proceed like in Refs. $[25,26]$; i.e., we find the corrections to the parameters of the theory by evaluating the former when a small change in the cutoff is applied. This physically corresponds to integrating out modes to keep the rescaled cutoff unchanged. In practice, we (i) use soft momentum cutoffs for the integrals, implemented by the use of a rapidly decaying function $|\mathbf{q}| / \Lambda \mapsto \mathcal{F}(|\mathbf{q}| / \Lambda)$, with, e.g., $\mathcal{F}$ belonging to the function space $\mathcal{L}^{2}(\mathbf{R})$; (ii) compute the logarithmic derivatives with respect to the cutoff $\Lambda$ of the fermion self-energy and vertices; (iii) identify the appropriate coefficients of the Taylor expansion (in $k_{i}$ and $\omega_{n}$ ) of the result. The choice of a soft cutoff is fairly arbitrary, but it helps to avoid spurious singularities induced by "ringing" at the spectral edge. The derivative with respect to $\Lambda$ serves to extract the incremental change in the band parameters due to a small change of cutoff, as in the Wilsonian view of RG. The momentum and frequency expansion allows identification of the renormalization of each term of the Hamiltonian independently.

\section{Diagram expressions}

The fermion self-energy is

$$
\Sigma_{f}\left(\omega_{n}, \mathbf{k}\right)=\sum_{b=\varphi, \phi} \frac{-g_{b}^{2}}{N} \int \frac{d^{3} q}{(2 \pi)^{3}} \int_{-\infty}^{+\infty} \frac{d \Omega_{n}}{(2 \pi)} \frac{M_{b} G_{\Omega_{n}, \mathbf{q}}^{0} M_{b} \mathcal{F}\left(\frac{|\mathbf{q}|}{\Lambda}\right) \mathcal{F}\left(\frac{|\mathbf{k}-\mathbf{q}|}{\Lambda}\right)}{\Sigma_{b}\left(\omega_{n}-\Omega_{n}, \mathbf{k}-\mathbf{q}\right)-\Sigma_{b}(0, \mathbf{0})},
$$

where two cutoff functions $\mathcal{F}$ are present because both fermion lines in the self-energy should be cut off; i.e., the momenta of all the electrons in the theory are taken within the cutoff. Similarly, the vertex corrections at zero external momenta and frequencies are $\Xi_{b}^{0}=\Xi_{b ;(1)}^{0}+\Xi_{b ;(2)}^{0}$, with

$$
\Xi_{b ;(1)}^{0}=\sum_{b^{\prime}=\varphi, \phi} \frac{g_{b} g_{b^{\prime}}^{2}}{N^{3 / 2}} \int \frac{d^{3} q}{(2 \pi)^{3}} \int_{-\infty}^{+\infty} \frac{d \Omega_{n}}{2 \pi} \frac{M_{b^{\prime}} G_{\Omega_{n}, \mathbf{q}}^{0} M_{b} G_{\Omega_{n}, \mathbf{q}}^{0} M_{b^{\prime}} \mathcal{F}^{2}\left(\frac{|\mathbf{q}|}{\Lambda}\right)}{\Sigma_{b^{\prime}}\left(\Omega_{n}, \mathbf{q}\right)-\Sigma_{b^{\prime}}(0, \mathbf{0})}
$$

and

$$
\begin{aligned}
\Xi_{b ;(2)}^{0}= & -\sum_{b^{\prime}, b^{\prime \prime}=\varphi, \phi ; \eta= \pm} \frac{g_{b} g_{b^{\prime}}^{2} g_{b^{\prime \prime}}^{2}}{N^{5 / 2}} \int \frac{d^{3} q_{1}}{(2 \pi)^{3}} \int \frac{d^{3} q_{2}}{(2 \pi)^{3}} \int_{-\infty}^{+\infty} \frac{d \Omega_{n, 1}}{2 \pi} \int_{-\infty}^{+\infty} \frac{d \Omega_{n, 2}}{2 \pi} \\
& \times \frac{M_{b^{\prime}} G_{\Omega_{n, 2}, \mathbf{q}_{2}}^{0} M_{b^{\prime \prime}} \operatorname{Tr}\left\{G_{\Omega_{n, 1}, \mathbf{q}_{1}}^{0} M_{b^{\prime}} G_{\Omega_{n, 1}+\eta \Omega_{n, 2}, \mathbf{q}_{1}+\eta \mathbf{q}_{2}}^{0} M_{b^{\prime \prime}} G_{\Omega_{n, 1}, \mathbf{q}_{1}}^{0} M_{b}\right\}}{\left[\Sigma_{b^{\prime}}\left(\Omega_{n, 2}, \mathbf{q}_{2}\right)-\Sigma_{b^{\prime}}(0, \mathbf{0})\right]\left[\Sigma_{b^{\prime \prime}}\left(\Omega_{n, 2}, \mathbf{q}_{2}\right)-\Sigma_{b^{\prime \prime}}(0, \mathbf{0})\right]} \mathcal{F}\left(\frac{\left|\mathbf{q}_{1}\right|}{\Lambda}\right) \mathcal{F}\left(\frac{\left|\mathbf{q}_{2}\right|}{\Lambda}\right) \mathcal{F}\left(\frac{\left|\mathbf{q}_{1}+\eta \mathbf{q}_{2}\right|}{\Lambda}\right) .
\end{aligned}
$$

All other diagrams are smaller in a $1 / N$ expansion. By using, for example, $\partial_{-i \alpha \omega_{n}} G_{\omega_{n}, \mathbf{k}}^{0}=-\left(G_{\omega_{n}, \mathbf{k}}^{0}\right)^{2}$, one can show that the two-loop diagrams $\Xi_{b ;(2) ; b^{\prime}, b^{\prime \prime}, \text {, }}^{0}$, with identical internal boson propagators $\left(b^{\prime}=b^{\prime \prime}\right)$, cancel each other out after performing the sum over $\eta= \pm 1$ (and even vanish identically in the case $b=\phi$ ). The remaining two-loop diagrams correcting the Coulomb vertex $(b=\varphi$ and $b^{\prime} \neq b^{\prime \prime}$ ) can also be shown to vanish, for example, by noticing that only the $b^{\prime}=b^{\prime \prime}$ diagrams can renormalize $g$. Therefore, only four two-loop diagrams (those with $b=\phi$ and $b^{\prime} \neq b^{\prime \prime}$ ), shown in Fig. 4 in the main text, need to be calculated. Careful observation shows that all contributions are equal, and an explicit calculation yields a finite integral, which converges to a nonzero value multiplied by $g /\left(N^{3 / 2}\left|\ln c_{1} / c_{2}\right|^{2}\right)$. This is actually subdominant (for $\left.c_{1} / c_{2} \ll 1\right)$ to the contribution from the one-loop vertex correction, although it is of the same order in $1 / N$.

\section{Flow equations}

We find the following RG flow equations ("beta functions"). The flow of $\alpha$, the coefficient of the frequency in the fermion self-energy, is

$$
\frac{\partial_{\ell} \alpha}{\alpha}=3-2 \Delta_{\psi}+\left.\frac{1}{\alpha}\left(\partial_{-i \omega_{n}}\left[D_{\Lambda} \Sigma_{f}^{0}\right]\right)\right|_{\omega_{n}=0, \mathbf{k}=\mathbf{0}}
$$

where $D_{\Lambda}=\Lambda(d / d \Lambda)$. As usual, the last term on the right-hand side corresponds, in the RG procedure, to the "rescaling" (or integration of momenta), while the other terms correspond to the "renormalization" [37]. The "anisotropic" coupling of the fermions to the bosons leads to "anisotropic" corrections to the coefficients of the fermion Hamiltonian: 


$$
\frac{\partial_{\ell} c_{j}}{c_{j}}=z+1-2 \Delta_{\psi}+\frac{\left(\delta \Sigma_{f}\right)_{j}^{0}}{c_{j}}, \quad j=0,1,2
$$

where

$$
\left(\delta \Sigma_{f}\right)_{j}^{0}= \begin{cases}\left.\frac{1}{2}\left(\partial_{k_{x}, k_{x}}^{2}\left[D_{\Lambda} \Sigma_{f}^{0}\right]\right)\right|_{\omega_{n}=0, \mathbf{k}=\mathbf{0}} & \text { for } j=0 \\ \left.\sqrt{2}\left(\partial_{k_{x}, k_{y}}^{2}\left[D_{\Lambda} \Sigma_{f}^{1}\right]\right)\right|_{\omega_{n}=0, \mathbf{k}=\mathbf{0}} & \text { for } j=1 \\ \left.\sqrt{2}\left(\partial_{k_{x}, k_{x}}^{2}\left[D_{\Lambda} \Sigma_{f}^{4}\right]\right)\right|_{\omega_{n}=0, \mathbf{k}=\mathbf{0}} & \text { for } j=2 .\end{cases}
$$

The RG equations for the coupling constants are simply

$$
\frac{\partial_{\ell} g_{b}}{g_{b}}=z+3-\Delta_{\phi}-2 \Delta_{\psi}+M_{b}^{-1} \frac{\left[D_{\Lambda} \Xi_{b}^{0}\right]}{g_{b} / \sqrt{N}},
$$

for $g_{\phi}=g, g_{\varphi}=i e$ and $M_{\phi}=\Gamma_{45}, M_{\varphi}=I$. The righthand sides of the equations eventually involve angular integrals that can be performed numerically and which are obtained using the identities

$$
\left\{\begin{array}{l}
\int_{0}^{\infty} d q \frac{1}{q} \Lambda \frac{d}{d \Lambda}\left[\mathcal{F}^{2}(q / \Lambda)\right]=1 \\
\int_{0}^{\infty} d q \Lambda \frac{d}{d \Lambda}\left[\frac{\mathcal{F}(q / \Lambda) \mathcal{F}^{\prime}(q / \Lambda)}{\Lambda}\right]=0 \\
\int_{0}^{\infty} d q q \Lambda \frac{d}{d \Lambda}\left[\frac{\mathcal{F}(q / \Lambda) \mathcal{F}^{\prime \prime}(q / \Lambda)}{\Lambda^{2}}\right]=0 \\
\int_{0}^{\infty} d q_{1} \frac{\Lambda}{q_{1}} \frac{d}{d \Lambda}\left[\mathcal{F}\left(q_{1} / \Lambda\right) \mathcal{F}\left(q_{1} \tilde{q}_{2} / \Lambda\right) \mathcal{F}\left(q_{1}\left(1+\tilde{q}_{2}\right) / \Lambda\right)\right]=1
\end{array}\right.
$$

(for any $\left.\tilde{q}_{2}\right)$, since $\mathcal{F}(0)=1$ and $\mathcal{F}(+\infty)=0 \quad\left(\mathcal{F}^{\prime}\right.$ and $\mathcal{F}^{\prime \prime}$ denote the first and second derivative of $\mathcal{F}$, respectively.).

In practice, to calculate the flows of $\alpha$ and $c_{0}$, from Eqs. (C4) and (C5) with $j=0$, we shift the internal momentum in the integrands of $\Sigma_{f}$ [see Eq. (C1)], i.e., $\mathbf{q} \rightarrow \mathbf{q}+\mathbf{k}$. As a result, the derivatives with respect to the frequency $\omega_{n}$ or momenta $k_{i}$ involve the fermionic part of the integrands. Proceeding otherwise to obtain the equation for $c_{0}$ leads to a divergent integral. For the flow of $c_{2}$, where the derivatives with respect to either part of the integral converge, we have checked that both "methods" give the same result. The integrals from the vertex corrections converge; in particular, we find that the double integrals in $\left[D_{\Lambda} \Xi_{b ;(2)}^{0}\right]$ are subdominant (equal to a finite number times $1 /\left|\ln c_{1} / c_{2}\right|^{2}$, the latter factor coming solely from the two inverse boson propagators), even upon taking $c_{0,1}=0$ directly in $G^{0}$.

\section{Details of the flows of $c_{1}$ and $c_{2}$}

Because the results are crucial to the physics, we give the details of the calculation of the beta functions for $c_{1}$ and $c_{2}$. Applying the derivatives in Eq. (C6) with $j=1,2$ to the "boson parts" of the integrand in the self-energies using the approximations discussed in Appendix $\mathrm{B}$, and expanding $\Sigma_{b}^{-1}$ in small $1 /\left|\ln c_{1} / c_{2}\right|$, we find

$$
\begin{aligned}
& \frac{\left(\delta \Sigma_{f}\right)_{1}^{0}}{c_{1}}=-\frac{\sqrt{2}}{8 \pi\left|\ln c_{1} / c_{2}\right| N} \int \frac{d \hat{\mathbf{q}}}{(2 \pi)^{2}} \frac{d_{1}(\hat{\mathbf{q}})}{E_{\hat{\mathbf{q}}}}\left\{\left(m_{1}^{\varphi}+m_{1}^{\phi}\right) \mathcal{N}_{1,1}+\left(m_{2}^{\varphi}+m_{2}^{\phi}\right) \mathcal{N}_{1,2}+\left(m_{3}^{\varphi}+m_{3}^{\phi}\right) \mathcal{N}_{1,3}\right\} \\
& \frac{\left(\delta \Sigma_{f}\right)_{2}^{0}}{c_{2}}=-\frac{\sqrt{2}}{8 \pi\left|\ln c_{1} / c_{2}\right| N} \int \frac{d \hat{\mathbf{q}}}{(2 \pi)^{2}} \frac{d_{4}(\hat{\mathbf{q}})}{E_{\hat{\mathbf{q}}}}\left\{\left(m_{1}^{\varphi}-m_{1}^{\phi}\right) \mathcal{N}_{2,1}+\left(m_{2}^{\varphi}-m_{2}^{\phi}\right) \mathcal{N}_{2,2}+\left(m_{3}^{\varphi}-m_{3}^{\phi}\right) \mathcal{N}_{2,3}\right\},
\end{aligned}
$$

where

$$
\begin{gathered}
\mathcal{N}_{1,1}=3 \hat{q}_{x} \hat{q}_{y} \\
\mathcal{N}_{1,2}=5 \hat{q}_{x} \hat{q}_{y}\left(-8 \hat{q}_{x}^{2} \hat{q}_{y}^{2}-4 \hat{q}_{x}^{2} \hat{q}_{z}^{2}-4 \hat{q}_{y}^{2} \hat{q}_{z}^{2}\right. \\
\left.+3 \hat{q}_{x}^{4}+3 \hat{q}_{y}^{4}+7 \hat{q}_{z}^{4}\right) \\
\mathcal{N}_{1,3}=-\hat{q}_{x} \hat{q}_{y} \hat{q}_{z}^{2}\left(6 \hat{q}_{x}^{2} \hat{q}_{z}^{2}-43 \hat{q}_{x}^{2} \hat{q}_{y}^{2}+6 \hat{q}_{y}^{2} \hat{q}_{z}^{2}\right. \\
\left.+10 \hat{q}_{x}^{4}+10 \hat{q}_{y}^{4}-4 \hat{q}_{z}^{4}\right) \\
\mathcal{N}_{2,1}=2 \hat{q}_{x}^{2}-\hat{q}_{y}^{2}-\hat{q}_{z}^{2} \\
\mathcal{N}_{2,2}=24 \hat{q}_{x}^{2} \hat{q}_{y}^{2} \hat{q}_{z}^{2}-21 \hat{q}_{x}^{4}\left(\hat{q}_{y}^{2}+\hat{q}_{z}^{2}\right)+\hat{q}_{y}^{4}\left(42 \hat{q}_{x}^{2}-5 \hat{q}_{z}^{2}\right) \\
+\hat{q}_{z}^{4}\left(42 \hat{q}_{x}^{2}-5 \hat{q}_{y}^{2}\right)+2 \hat{q}_{x}^{6}-5 \hat{q}_{y}^{6}-5 \hat{q}_{z}^{6}
\end{gathered}
$$

$$
\begin{aligned}
\mathcal{N}_{2,3}= & \hat{q}_{y}^{2} \hat{q}_{z}^{2}\left(-31 \hat{q}_{x}^{2} \hat{q}_{y}^{2}-31 \hat{q}_{x}^{2} \hat{q}_{z}^{2}+4 \hat{q}_{y}^{2} \hat{q}_{z}^{2}\right. \\
& \left.+30 \hat{q}_{x}^{4}+2 \hat{q}_{y}^{4}+2 \hat{q}_{z}^{4}\right) .
\end{aligned}
$$

The relative signs of the terms coming from $\Sigma_{\phi}$ originate from the "opposite" commutation relations of $\Gamma_{1,2,3}$ and $\Gamma_{4,5}$ with $\Gamma_{45}$, i.e., $\left[\Gamma_{a}, \Gamma_{45}\right]=0$ for $a=1,2,3$ and $\left\{\Gamma_{a}, \Gamma_{45}\right\}=0$ for $a=4,5$. Note that this is true before implementing any approximation or assumption on the magnitude of $c_{1} / c_{2}$. If $e=0$, it is then obvious that the flows of $c_{1}$ and $c_{2}$ will take different directions, i.e., that the ratio $c_{1} / c_{2}$ will be either relevant or irrelevant or, in other words, that this ratio will flow either to infinity or zero. Hence, a calculation taking $c_{1} / c_{2}$ large or small from the beginning is definitely valid. We find that $c_{1} / c_{2} \rightarrow 0$ occurs for $e=0$ (see below). When $e \neq 0$, the situation is 
not as clear-cut, but taking $c_{1} / c_{2}$ small, as when $e=0$, proves to be self-consistent, as shown below. We can also justify it a posteriori as follows. $c_{1} / c_{2} \rightarrow+\infty$ would lead to a situation where the coupling term $\phi \psi^{\dagger} \Gamma_{45} \psi$ commutes with the bare Hamiltonian at the critical point, hence removing all fluctuations due to the coupling to the order parameter, which is supposed to drive the transition through the fluctuations it induces. Such a choice seems therefore unreasonable. The situation where $c_{1} / c_{2} \rightarrow c^{*}$, a fixed constant, although perhaps seemingly more reasonable, would imply the existence of a universal ratio, when none seems to be natural. Hence, the limit $c_{1} / c_{2} \rightarrow 0$ seems to be the only reasonable limit to be taken. $c_{0} / c_{1} \rightarrow 0$ is also consistent.

\section{Exponents}

Keeping $\alpha, c_{2}, g$, and $e$ constant, i.e., setting the corresponding flow equations to zero, the dynamical critical exponent and the field dimensions are

$$
\begin{aligned}
z & =2-\frac{a_{z}}{N\left|\ln c_{1} / c_{2}\right|}, \quad \Delta_{\psi}=\frac{3}{2}+\frac{a_{\psi}}{N\left|\ln c_{1} / c_{2}\right|^{2}}, \\
\Delta_{\phi} & =\frac{3}{2}+\left[\frac{1}{2}+\frac{a_{\phi}}{N\left|\ln c_{1} / c_{2}\right|}\right], \\
\Delta_{\varphi} & =\frac{3}{2}+\left[\frac{1}{2}-\frac{a_{\varphi}}{N\left|\ln c_{1} / c_{2}\right|}\right],
\end{aligned}
$$

where $a_{z}=0.063, \quad a_{\psi}=0.143, \quad a_{\phi}=0.255, \quad$ and $a_{\varphi}=0.063$. The anomalous dimensions are then simply $\delta z=a_{z} /\left(N\left|\ln c_{1} / c_{2}\right|\right), \quad \eta_{\psi}=2 a_{\psi} /\left(N\left|\ln c_{1} / c_{2}\right|^{2}\right), \quad \eta_{\phi}=$ $1+2 a_{\phi} /\left(N\left|\ln c_{1} / c_{2}\right|\right)$, and $\eta_{\varphi}=1-2 a_{\varphi} /\left(N\left|\ln c_{1} / c_{2}\right|\right)$, as given in the main text.

\section{Solutions to the flow equations}

Finally, we obtain

$$
\begin{aligned}
& \partial_{\ell}\left(\frac{c_{1}}{c_{2}}\right)=-\frac{c_{1}}{c_{2}} \frac{Y}{N\left|\ln c_{1} / c_{2}\right|}, \\
& \partial_{\ell}\left(\frac{c_{0}}{c_{1}}\right)=-\frac{c_{0}}{c_{1}} \frac{W}{N\left|\ln c_{1} / c_{2}\right|},
\end{aligned}
$$

with $Y=0.020$ and $W=0.043$. These equations are solved analytically by

$$
\begin{aligned}
& \left(c_{1} / c_{2}\right)(\ell)=e^{-\frac{v_{0}}{\sqrt{N}} \sqrt{\ell+\ell_{0}}}, \\
& \left(c_{0} / c_{1}\right)(\ell)=\Upsilon_{0} e^{-\frac{v_{0}{ }^{\prime}}{\sqrt{N}} \sqrt{\ell+\ell_{0}}}
\end{aligned}
$$

where $v_{0}=\sqrt{2 Y}$ and $v_{0}{ }^{\prime}=\sqrt{2} W / \sqrt{Y}$, and where $\ell_{0}$ and $\Upsilon_{0}$ are constants that depend on $c_{0,1,2}(\ell=0)$.

Note that, as mentioned in the main text, in the absence of Coulomb interactions, we find $\left(c_{1} / c_{2}\right)(\ell)=$ $e^{-(0.359 / \sqrt{N}) \sqrt{\ell+\ell_{1}}}$ and $\left(c_{0} / c_{1}\right)(\ell) \propto e^{(0.240 / \sqrt{N}) \sqrt{\ell+\ell_{1}}}$
( $\ell_{1}$ is a constant); i.e., $c_{0} / c_{1}$ is found to be a relevant parameter in that case. The latter means that, eventually, $c_{0}$ reaches $c_{1} / \sqrt{6}$, a point at which Fermi surfaces start to develop, rendering our theory invalid and the heretofore studied critical point unstable. This would correspond to a Lifshitz transition.

\section{APPENDIX D: PHYSICAL QUANTITIES}

We are now in a position to calculate the behavior of some physical quantities. We first extract the critical exponent of the correlation length. The associated RG flow is

$$
\frac{\partial_{\ell} r}{r}=z+3-2 \Delta_{\phi}, \quad \text { i.e., } \quad \partial_{\ell} r=\nu^{-1}(\ell) r,
$$

with

$$
\nu^{-1}(\ell)=1-\frac{2 a_{\phi}+a_{z}}{N\left|\ln c_{1} / c_{2}\right|}
$$

So

$$
\partial_{\ell} r=\left(1-\frac{A}{\sqrt{N} \sqrt{\ell+\ell_{0}}}\right) r, \quad \text { with } \quad A=\frac{2 a_{\phi}+a_{z}}{v_{0}},
$$

i.e., $A=2.836$, which is solved as

$$
r(\ell)=r_{0} e^{\ell-(2 A / \sqrt{N}) \sqrt{\ell+\ell_{0}}}
$$

where $r_{0}$ is a constant that depends on $r(\ell=0)$. We can easily invert $r=r(\ell)$ to $\ell=\ell(r)$ by taking the log of Eq. (D4), squaring both sides, and solving the quadratic equation. We get

$$
\ell \approx \ln \frac{r}{r_{0}}+\frac{2 A}{\sqrt{N}} \sqrt{\ln \frac{r}{r_{0}}+\ell_{0}},
$$

where we have kept only terms to order $1 / \sqrt{N}$.

\section{Order-parameter exponent}

We first extract the exponent $\beta$ and its logarithmic correction, i.e., how $\langle\phi\rangle$ behaves with $r$. We write

$$
\frac{\phi(\ell+d \ell)-\phi(\ell)}{\phi(\ell+d \ell)} \approx d \ell \Delta_{\phi}(\ell)
$$

and integrate both sides from 0 to $\ell$. Using Eq. (D5), we obtain

$$
\begin{aligned}
\frac{\phi}{\phi_{0}} \sim & \left(\frac{r}{r_{0}}\right)^{2} \exp \left[2 \frac{5 a_{\phi}+2 a_{z}}{v_{0} \sqrt{N}} \sqrt{\ln \frac{r}{r_{0}}+\ell_{0}}\right] \\
& \times \exp \left[\frac{-2 a_{\phi} \sqrt{\ell_{0}}}{v_{0} \sqrt{N}}\right]
\end{aligned}
$$


with $2\left(5 a_{\phi}+2 a_{z}\right) / v_{0}=13.867$ and $2 a_{\phi} / v_{0}=2.523$ (in the main text, we absorbed $\ell_{0}$ in the definition of $r_{0}$ ). Contrary to more conventional problems, like the usual Ising model, where $\beta_{\text {Ising }}=1 / 2$ in three spatial dimensions, the bosonic order parameter here grows very slowly as one moves away from the critical point on the ordered side of the transition. This comes from the massive fluctuations of the boson field due to the strong coupling to the fermions.

\section{Specific heat}

At the critical point (or in the quantum critical region), temperature is the only relevant parameter, so thermal properties receive intriguing corrections in our critical theory. Since the fermion is well defined $\left(\eta_{\psi} \rightarrow 0\right)$, the thermal average of the energy is

$$
\langle\mathrm{E}\rangle=\sum_{i= \pm, \mathbf{k}}\left\langle n_{i, \mathbf{k}}\right\rangle \mathrm{E}_{i}(\mathbf{k})=\sum_{i, \mathbf{k}} \frac{2}{e^{\beta \mathrm{E}_{i}(\mathbf{k})}+1} \mathrm{E}_{i}(\mathbf{k}),
$$

with, for $\langle\phi\rangle=0, \quad c_{0}=0 \quad$ and $\quad c_{2}=1, \quad \mathrm{E}_{ \pm}(\mathbf{k})=$ $\pm\left(\mathbf{k}^{2} / \sqrt{6}\right) \sqrt{1+3\left(c_{1}^{2}-1\right) w_{\hat{\mathbf{k}}}^{2}}$, where $w_{\hat{\mathbf{k}}}=\hat{k}_{x}^{2} \hat{k}_{y}^{2}+\hat{k}_{x}^{2} \hat{k}_{z}^{2}+$ $\hat{k}_{y}^{2} \hat{k}_{z}^{2}$. To lowest order, we find

$$
\begin{aligned}
c_{V}=\partial_{T}\langle\mathrm{E}\rangle & \approx \frac{15(4-\sqrt{2}) 6^{3 / 4} \sqrt{\pi}}{16} \zeta(5 / 2) \frac{T^{3 / 2}}{c_{1}^{3 / 2}} \\
& \approx 22.1 \exp \left[\frac{3 v_{0}}{2 \sqrt{N} \sqrt{z}} \sqrt{\ln \frac{T_{0}}{T}}\right] T^{3 / 2},
\end{aligned}
$$

where $3 v_{0} /(2 \sqrt{z}) \approx 0.215$ (we use $z \approx 2$ ) [ $\zeta$ is the Riemann zeta function.]. To obtain the last line, we used the approximation $e^{\ell}=\left(T(\ell) / T_{0}\right)^{1 / z}$ and thereby solved the $\mathrm{RG}$ equation of $c_{1}$ in terms of temperature. The logarithmic correction to the $T^{3 / 2}$ law is a signature of the fact that $c_{1}$ becomes scale (temperature) dependent in the quantum critical region.

\section{APPENDIX E: MEAN-FIELD THEORY}

In this appendix, we consider the behavior in the ordered phase according to naïve mean-field theory, i.e., a saddlepoint evaluation of the $\varphi$ and $\phi$ integrals. The former saddle point is simply $\varphi=0$; i.e., there are no effects of the longrange Coulomb interactions at the mean-field level. The saddle-point value of $\phi$ is nonzero in the antiferromagnetic phase. It is governed by the effective action that consists of the bare one [second line of Eq. (2) of the main text] plus the contribution obtained by integrating out the fermions.

The fermionic contribution to the effective action, for constant $\phi$, is simply the space-time integral of the total ground-state energy density of the electrons. This is obtained by summing up the energy of occupied singleparticle states.

In the saddle-point approximation, the Hamiltonian density of the fermions is

$$
\mathcal{H}_{\mathrm{MF}}^{\psi}[\phi]=c_{0} \mathbf{k}^{2}+\sum_{a=1}^{5} \hat{c}_{a} d_{a}(\mathbf{k}) \Gamma_{a}+\phi \Gamma_{45},
$$

and we therefore have the ground-state energy density

$$
\mathcal{E}^{\psi}{ }_{\mathrm{MF}}[\phi]=\sum_{\alpha=1}^{2} \int_{\Lambda} \frac{d^{3} k}{(2 \pi)^{3}} \mathrm{E}_{\mathbf{k}}^{\alpha}[\phi]
$$

$\left[E_{\mathbf{k}}^{1,2}\right.$ are the single-particle lowest-energy bands, with $\left.\mathrm{E}_{\mathbf{k}}^{1,2}[\phi=0]=\mathrm{E}_{-}(\mathbf{k})\right]$. Here, by diagonalizing $\mathcal{H}_{\mathrm{MF}}^{\psi}[\phi]$, we obtain

$$
\mathrm{E}_{\mathbf{k}}^{1,2,3,4}[\phi]=c_{0} \mathbf{k}^{2} \pm \frac{1}{\sqrt{6}} \sqrt{c_{2}^{2} \mathbf{k}^{4}+2\left(c_{1}^{2}-c_{2}^{2}\right) w_{\hat{\mathbf{k}}}^{2} \mathbf{k}^{4} \pm 6 \sqrt{2} c_{1} \mathbf{k}^{2} w_{\hat{\mathbf{k}}} \phi+6 \phi^{2}}
$$

where we define $\mathbf{k}^{4}=\left(\mathbf{k}^{2}\right)^{2}$ and where 1,2,3,4 correspond to the signs $\{--,-+,+-,++\}$, respectively.

From scaling, $E_{\mathbf{k}}^{1,2} \sim \mathbf{k}^{2}$, and hence, from Eq. (E2), one expects that the singular scaling contributions to the effective action behave as $\mathcal{E}_{\mathrm{MF}}^{\psi} \sim|\mathbf{k}|^{5} \sim|\phi|^{5 / 2}$, where we used $\phi \sim \mathbf{k}^{2}$, which follows dimensionally from $\mathcal{H}_{\mathrm{MF}}^{\psi}$. This describes only the singular contributions. Since $\mathcal{E}_{\mathrm{MF}}^{\psi}$ is an even function of $\phi$, we expect it to contain constant and quadratic terms as well (which are cutoff dependent). Indeed, one can verify by direct expansion in $\phi$ that the integrals which arise from Eq. (E2) as coefficients of unity and $\phi^{2}$ are finite, but if one proceeds to the following order, the coefficient of $\phi^{4}$ is divergent. This is because of the presence of the $t|\phi|^{5 / 2}$ term.
To extract the coefficient $t$, we take three derivatives of $\mathcal{E}_{\mathrm{MF}}^{\psi}[\phi]$ with respect to $\phi$. We find an integral whose integrand goes as $1 /|\mathbf{k}|^{6}$ at large $|\mathbf{k}|$ so that the result is integrable in that region. One then simply rescales $\mathbf{k} \rightarrow$ $\mathbf{k} / \sqrt{|\phi|}$ and takes the limit of small $\phi$ (i.e., $\Lambda / \sqrt{\phi} \rightarrow+\infty$ ). This makes the singular behavior explicit, and in this limit, we find $\partial_{\phi, \phi, \phi}^{3} \mathcal{E}_{\mathrm{MF}}^{\psi}[\phi]=1.079 / \sqrt{\phi}$, i.e., $\quad t=$ $1.079 \times(8 / 15)=0.575$, where the coefficient was determined by a numerical integration, taking the fixed-point values $c_{0}=c_{1}=0$. Therefore, $t|\phi|^{5 / 2}$ is indeed the lowestorder nonanalytic term. Hence, putting everything together, and looking at the boson action with the fermions integrated out, we have 


$$
\begin{aligned}
\mathcal{S}_{\mathrm{MF}}[\phi] & =V \int d \tau\left[r \phi^{2}+\mathcal{E}_{\mathrm{MF}}^{\psi}[\phi]\right] \\
& \sim V \int d \tau\left[r^{\prime} \phi^{2}+t|\phi|^{5 / 2}\right],
\end{aligned}
$$

all other terms being irrelevant. Above, $r^{\prime}$ includes the $\phi^{2}$ terms in $\mathcal{E}_{\mathrm{MF}}^{\psi}[\phi]$. Most importantly, we obtained positive $t>0$ so that when $r^{\prime}<0$, a stable minimum action configuration exists, describing a continuous-but unconventional-transition at the mean-field level.

[1] S. Sachdev, Quantum Phase Transitions (Cambridge University Press, Cambridge, England, 2011).

[2] H. v. Löhneysen, A. Rosch, M. Vojta, and P. Wölfle, Fermi-Liquid Instabilities at Magnetic Quantum Phase Transitions, Rev. Mod. Phys. 79, 1015 (2007).

[3] J. A. Hertz, Quantum Critical Phenomena, Phys. Rev. B 14, 1165 (1976).

[4] A. J. Millis, Effect of a Nonzero Temperature on Quantum Critical Points in Itinerant Fermion Systems, Phys. Rev. B 48, 7183 (1993).

[5] S.-S. Lee, Low-Energy Effective Theory of Fermi Surface Coupled with $U(1)$ Gauge Field in $2+1$ Dimensions, Phys. Rev. B 80, 165102 (2009).

[6] M. A. Metlitski and S. Sachdev, Quantum Phase Transitions of Metals in Two Spatial Dimensions. II. Spin Density Wave Order, Phys. Rev. B 82, 075128 (2010).

[7] D. F. Mross, J. McGreevy, H. Liu, and T. Senthil, Controlled Expansion for Certain Non-Fermi-Liquid Metals, Phys. Rev. B 82, 045121 (2010).

[8] K. B. Efetov, H. Meier, and C. Pépin, Pseudogap State Near a Quantum Critical Point, Nat. Phys. 9, 442 (2013).

[9] X. Wan, A. M. Turner, A. Vishwanath, and S. Y. Savrasov, Topological Semimetal and Fermi-Arc Surface States in the Electronic Structure of Pyrochlore Iridates, Phys. Rev. B 83, 205101 (2011).

[10] E.-G. Moon, C. Xu, Y. Baek Kim, and L. Balents, Non-Fermi-Liquid and Topological States with Strong Spin-Orbit Coupling, Phys. Rev. Lett. 111, 206401 (2013).

[11] R. Chen, E.-G. Moon, and L. Balents (unpublished).

[12] T. Kondo, S. Nakatsuji et al. (unpublished).

[13] K. Matsuhira, M. Wakeshima, Y. Hinatsu, and S. Takagi, Metal-Insulator Transitions in Pyrochlore Oxides $\mathrm{Ln}_{2} \mathrm{Ir}_{2} \mathrm{O}_{7}$, J. Phys. Soc. Jpn. 80, 094701 (2011).

[14] W. Witczak-Krempa, A. Go, and Y. Baek Kim, Pyrochlore Electrons under Pressure, Heat, and Field: Shedding Light on the Iridates, Phys. Rev. B 87, 155101 (2013).

[15] H. Sagayama, D. Uematsu, T. Arima, K. Sugimoto, J. J. Ishikawa, E. O'Farrell, and S. Nakatsuji, Determination of Long-Range All-In-All-Out Ordering of $\mathrm{Ir}^{4+}$ Moments in a Pyrochlore Iridate $\mathrm{Eu}_{2} \mathrm{Ir}_{2} \mathrm{O}_{7}$ by Resonant X-Ray Diffraction, Phys. Rev. B 87, 100403 (2013).

[16] K. Tomiyasu, K. Matsuhira, K. Iwasa, M. Watahiki, S. Takagi, M. Wakeshima, Y. Hinatsu, M. Yokoyama, K. Ohoyama, and K. Yamada, Emergence of Magnetic Long-Range Order in Frustrated Pyrochlore $\mathrm{Nd}_{2} \mathrm{Ir}_{2} \mathrm{O}_{7}$ with
Metal-Insulator Transition, J. Phys. Soc. Jpn. 81, 034709 (2012).

[17] S. M. Disseler, C. Dhital, A. Amato, S. R. Giblin, C. de la Cruz, S. D. Wilson, and M. J. Graf, Magnetic Order in the Pyrochlore Iridates $\mathrm{A}_{2} \mathrm{Ir}_{2} \mathrm{O}_{7}(A=Y, Y b)$, Phys. Rev. B 86, 014428 (2012).

[18] K. A. Ross, L. Savary, B. D. Gaulin, and L. Balents, Quantum Excitations in Quantum Spin Ice, Phys. Rev. X 1, 021002 (2011).

[19] Q. Si and E. Abrahams, Strong Correlations and Magnetic Frustration in the High $T_{c}$ Iron Pnictides, Phys. Rev. Lett. 101, 076401 (2008).

[20] J. Kim, A. H. Said, D. Casa, M. H. Upton, T. Gog, M. Daghofer, G. Jackeli, J. van den Brink, G. Khaliullin, and B. J. Kim, Large Spin-Wave Energy Gap in the Bilayer Iridate $\mathrm{Sr}_{3} \mathrm{Ir}_{2} \mathrm{O}_{7}$ : Evidence for Enhanced Dipolar Interactions Near the Mott Metal-Insulator Transition, Phys. Rev. Lett. 109, 157402 (2012).

[21] J. W. Kim, Y. Choi, J. Kim, J. F. Mitchell, G. Jackeli, M. Daghofer, J. van den Brink, G. Khaliullin, and B. J. Kim, Dimensionality Driven Spin-Flop Transition in Layered Iridates, Phys. Rev. Lett. 109, 037204 (2012).

[22] A. A. Abrikosov and S. D. Beneslavskii, Possible Existence of Substances Intermediate between Metals and Dielectrics, Sov. Phys. JETP 32, 699 (1971).

[23] A. A. Abrikosov, Calculation of Critical Indices for Zero-Gap Semiconductors, Sov. Phys. JETP 39, 709 (1974).

[24] O. Vafek and A. Vishwanath, Dirac Fermions in Solids: From High- $T_{c}$ Cuprates and Graphene to Topological Insulators and Weyl Semimetals, Annu. Rev. Condens. Matter Phys. 5, 83 (2014).

[25] Y. Huh and S. Sachdev, Renormalization Group Theory of Nematic Ordering in d-Wave Superconductors, Phys. Rev. B 78, 064512 (2008).

[26] M. Vojta, Y. Zhang, and S. Sachdev, Renormalization Group Analysis of Quantum Critical Points in $d$-Wave Superconductors, Int. J. Mod. Phys. B 14, 3719 (2000).

[27] Note that a number of technicalities are involved in this calculation, in particular, regarding the convergence of the differentiated functions. They are all discussed in Appendices $\mathrm{C} 2$ and $\mathrm{C} 3$

[28] M. Kurita, Y. Yamaji, and M. Imada, Phase Diagram Structure of Topological Mott Transition for Zero-Gap Semiconductors beyond Conventional Landau-GinzburgWilson Scenario, Phys. Rev. B 88, 115143 (2013).

[29] W. Witczak-Krempa and Y. Baek Kim, Topological and Magnetic Phases of Interacting Electrons in the Pyrochlore Iridates, Phys. Rev. B 85, 045124 (2012).

[30] G. Chen and M. Hermele, Magnetic Orders and Topological Phases from $f$-d Exchange in Pyrochlore Iridates, Phys. Rev. B 86, 235129 (2012).

[31] R. Flint and T. Senthil, Chiral RKKY Interaction in $\mathrm{Pr}_{2} \mathrm{Ir}_{2} \mathrm{O}_{7}$, Phys. Rev. B 87, 125147 (2013).

[32] S. Lee, A. Paramekanti, and Y. Baek Kim, RKKY Interactions and the Anomalous Hall Effect in Metallic Rare-Earth Pyrochlores, Phys. Rev. Lett. 111, 196601 (2013).

[33] T. Hahn, Cuba-A Library for Multidimensional Numerical Integration, Comput. Phys. Commun. 168, 78 (2005). 
[34] D. Binosi and L. Theul, JaxoDraw: A Graphical User Interface for Drawing Feynman Diagrams, Comput. Phys. Commun. 161, 76 (2004).

[35] J. M. Luttinger, Quantum Theory of Cyclotron Resonance in Semiconductors: General Theory, Phys. Rev. 102, 1030 (1956).
[36] S. Murakami, N. Nagaosa, and S.-C. Zhang, SU(2) Non-Abelian Holonomy and Dissipationless Spin Current in Semiconductors, Phys. Rev. B 69, 235206 (2004).

[37] M. Kardar, Statistical Physics of Fields (Cambridge University Press, Cambridge, England, 2007). 